\title{
Decoherence-free radio-frequency-dressed subspaces
}

\author{
G. A. Sinuco-Leon $\odot,{ }^{1, *}$ H. Mas $\odot,{ }^{2,3, \dagger}$ S. Pandey $\odot,{ }^{2,4, \ddagger}$ G. Vasilakis, ${ }^{2}$ B. M. Garraway $\odot,{ }^{1}$ and W. von Klitzing ${ }^{2}$ \\ ${ }^{1}$ Department of Physics \& Astronomy, University of Sussex, Falmer, Brighton BN1 9QH, United Kingdom \\ ${ }^{2}$ Institute of Electronic Structure and Laser, Foundation for Research and Technology-Hellas, Heraklion 70013, Greece \\ ${ }^{3}$ Department of Physics, University of Crete, Heraklion 70013, Greece \\ ${ }^{4}$ Department of Materials, Science and Technology, University of Crete, Heraklion 70013, Greece
}

(Received 22 April 2021; accepted 28 June 2021; published 7 September 2021)

\begin{abstract}
We study the spectral signatures and coherence properties of radio-frequency-dressed hyperfine Zeeman sublevels of ${ }^{87} \mathrm{Rb}$. Experimentally, we engineer combinations of static and RF magnetic fields to modify the response of the atomic spin states to environmental magnetic field noise. We demonstrate analytically and experimentally the existence of "magic" dressing conditions where decoherence due to electromagnetic field noise is strongly suppressed. Building on this result, we propose a bichromatic dressing configuration that reduces the global sensitivity of the atomic ground states to low-frequency noise, and enables the simultaneous protection of multiple transitions between the two ground hyperfine manifolds of atomic alkali species. Our methods produce protected transitions between any pair of hyperfine sublevels at arbitrary (low) DC-magnetic fields.
\end{abstract}

DOI: 10.1103/PhysRevA.104.033307

\section{INTRODUCTION}

The sensitivity to environmental field fluctuations of atomic transitions in quantum systems represents a major challenge for improving the accuracy and reliability of applications such as atomic clocks [1], low-frequency field sensing [2], quantum memories [3] and information processors [4-6]. The sensitivity problem arises since, typically, the transition frequency between pairs of quantum states is affected by temporal and spatial variations of the electromagnetic environment, which results in a rapid dephasing of the system's wave function. The ubiquity of this problem, identified in ultracold atomic ensembles [7], superconducting devices [8], nitrogen-vacancy centers in diamond [9], and doped silicon [10], has driven the development of passive and active stabilization techniques for magnetic field shielding (e.g., Refs. [11,12]) as well as various flavors of dynamical coherent control (pulsed [13], concatenated [14], and continuous $[15,16])$ that isolate such systems from unwanted noise sources and improve their coherence time by several orders of magnitude [17]. In atomic systems, in particular, reduction of

\footnotetext{
*Present address: Department of Chemistry, Durham University, Durham DH1 3LE, UK.

${ }^{\dagger}$ Present address: Physics Department, University of California, Santa Barbara, CA 93106, USA.

${ }^{\ddagger}$ Present address: MPA Division, Los Alamos National Laboratory, Los Alamos, NM 87545, USA.

Published by the American Physical Society under the terms of the Creative Commons Attribution 4.0 International license. Further distribution of this work must maintain attribution to the author(s) and the published article's title, journal citation, and DOI.
}

the frequency broadening can be achieved partially by choosing appropriate states, which are less magnetically sensitive. However, often it is necessary to apply additional electromagnetic fields (static magnetic field (DC) [18], microwaves (MW) [19], radio-frequency (RF) [20], optical radiation [21]) tuned to suppress the differential Zeeman or Stark energy shifts between selected pairs of states (clock/qubit states), which, effectively, protects the transition frequency against field fluctuations.

Here we demonstrate the control of the magnetic field sensitivity of the electronic ground state of ${ }^{87} \mathrm{Rb}$, dressed by a radio-frequency magnetic field. The dressing is achieved by applying an oscillating magnetic field, $\boldsymbol{B}_{\mathrm{RF}}$, with frequency, $\omega_{\mathrm{RF}}$, close to the Larmor frequency (or Zeeman splitting) and controlled by an applied static magnetic field, $\boldsymbol{B}_{\mathrm{DC}}$. We use microwave spectroscopy $[22,23]$ to determine the energy difference and linewidth of transitions between dressed states $[24,25]$ and study their dependence with respect to the dressing configuration. We observe that, in general, for any pair of dressed states there is a dressing condition for which the atoms decouple from fluctuations of the static field, which results in a significant reduction of the line broadening. In our setup, using a linearly polarized RF dressing field, we find that the broadening of dressed transitions lines $(\sim 0.1 \mathrm{kHz})$ is one order of magnitude smaller than that of equivalent bare transitions $(\sim 1.0 \mathrm{kHz})$, limited mainly by amplitude noise of our RF generator [26]. We also find that the optimal dressing condition (i.e., that with reduced magnetic field sensitivity) depends on the selected pair of dressed states as a result of the difference of the gyromagnetic factors of the two electronic ground-state hyperfine manifolds, nonlinear Zeeman shifts [27] and Bloch-Siegert shift effects [28].

The majority of existing techniques for noise suppression focus on qubit and clock applications. There is, however, 
a growing interest in manipulating qudit systems in atomic [29,30] and solid-state [31] platforms, which requires the control of $d$ internal states [32-34]. In this paper, we propose a dressing scheme that reduces the sensitivity of all possible hyperfine transitions in the electronic ground state of ${ }^{87} \mathrm{Rb}$. Our scheme exploits the possibility of addressing independently each hyperfine manifold by tuning the frequency of each circular component $\left(\sigma_{ \pm}\right)$of the dressing field [35].

As a figure of merit in the improvement in the stability against low-frequency noise, and thus of the atomic resilience to decoherence [36,37], we evaluate the root-mean-square (rms) average of the first derivative of the atomic transitions frequencies with respect to static field, $\alpha_{\mathrm{DC}}$ (see Sec. IV). Using the rotating wave approximation (RWA) and neglecting the quadratic Zeeman shift, we found a first estimate of the ratio between the $\sigma_{ \pm}$frequencies that minimizes $\alpha_{\mathrm{DC}}$. This optimal condition should be corrected to take into account effects from nonlinear Zeeman and Bloch-Siegert shifts, which we explain qualitatively and evaluate numerically. We found that, although it is not possible to fully cancel the influence of magnetic field fluctuations for all transitions, our scheme defines dressed states with a magnetic sensitivity smaller than that possible with bare and single-frequency-dressed atoms. This scheme can be used to improve the robustness of qudits encoded in the electronic ground state of alkali atoms, for which control protocols have been recently demonstrated using microwave pulses [29,38].

The structure of this paper is as follows. Section II reviews the formalism we use to define the basis of dressed states. In Sec. III we present experimental results for the dependence of the transition frequencies between dressed states with respect to the applied static magnetic field, which reflects into a dependence of the linewidth and decoherence. Following this, in Sec. IV we propose a bichromatic dressing configuration that leads to an overall reduction of the magnetic sensitivity (on average) of all possible transitions between dressed states. The closing section (Sec. V) presents the conclusions of our work.

\section{RF DRESSING OF THE GROUND-STATE MANIFOLD OF ALKALI ATOMS}

The internal dynamics of an alkali atom in its electronic ground state interacting with a magnetic field, $\boldsymbol{B}(t)$, is governed by the Hamiltonian:

$$
H(t)=\frac{A}{\hbar^{2}} \boldsymbol{I} \cdot \boldsymbol{J}+\frac{\mu_{B}}{\hbar}\left(g_{I} \boldsymbol{I}+g_{J} \boldsymbol{J}\right) \cdot \boldsymbol{B}(t),
$$

where $A$ is the hyperfine coupling, $\mu_{B}$ is the Bohr magneton, and the factors $g_{I}$ and $g_{J}$ are the nuclear $(\boldsymbol{I})$ and electronic $(\boldsymbol{J})$ Landé $g$-factors, with corresponding angular momentum operators $\boldsymbol{I}$ and $\boldsymbol{J}$. The coupling between nuclear and electronic magnetic momenta defines two hyperfine manifolds (given $J=1 / 2$ ) with different total angular momentum, $F=I \pm$ $1 / 2$, which are split by an energy gap of $\hbar \omega_{\mathrm{hfs}}=A(I+1 / 2)$. This splitting defines a natural basis of states to describe the atomic dynamics, labeled by the total angular momentum $(F)$ and its projection along a quantization axis $(m),\{|F, m\rangle\}$.
Here we study RF-dressed atoms of ${ }^{87} \mathrm{Rb}$ prepared by the magnetic field:

$$
\boldsymbol{B}(t)=B_{\mathrm{DC}} \hat{\boldsymbol{e}}_{z}+\sum_{i \in\{x, y, z\}} B_{\mathrm{RF}, i} \cos \left(\omega_{\mathrm{RF}} t+\phi_{i}\right) \hat{\boldsymbol{e}}_{i},
$$

where the quantization axis $z$ is defined along the direction of the static field $\boldsymbol{B}_{\text {DC }}$.

The dressed basis is defined as the set of solutions of the Schrödinger equation resulting from Eqs. (1) and (2), with the form:

$$
\left|\Psi_{F \bar{m}}\right\rangle=e^{-i \bar{E}_{F, \bar{m}} t / \hbar}|F, \bar{m}\rangle,
$$

where $\bar{E}_{F, \bar{m}}$ is the corresponding dressed energy [39]. The ket on the right-hand side of Eq. (3) is a time-periodic linear combination of the bare states, which can be expressed as the Fourier series:

$$
|F, \bar{m}\rangle=\sum_{m} \sum_{n} U_{m, \bar{m}}^{n} e^{i n \omega_{\mathrm{RF}} t}|F, m\rangle .
$$

The existence of this solution of the Schrödinger equation is guaranteed by the Floquet-Bloch theorem [40].

In our experiments, see Sec. III, we transform the eigenstates of the static Hamiltonian, $\{|F, m\rangle\}$ into the dressed states $\{|F, \bar{m}\rangle\}$, by adiabatically varying the DC and RF fields until reaching a final dressing configuration. We achieve highfidelity transformations from the bare to the dressed basis by defining temporal trajectories of the field configuration that avoid the degeneracy of the dressed energies $\left\{\bar{E}_{F, \bar{m}}\right\}$ [40]. Furthermore, the absence of level crossings during the switchover to the dressed basis allows us to establish a one-to-one correspondence between the bare and the dressed basis, such that we can use the same set of quantum numbers for labeling the two bases.

In the limit of weak dressing and linear Zeeman shift, the Fourier coefficients in Eq. (4) can be approximated using the RWA with the dressed energy [41] (see Appendix A):

$$
\bar{E}_{F, \bar{m}}=E_{F}+\frac{g_{F}}{\left|g_{F}\right|} \bar{m} \sqrt{\left[\hbar \omega_{0}-\hbar \omega_{\mathrm{RF}}^{\mathrm{sgn}\left(g_{F}\right)}\right]^{2}+2\left|\hbar \Omega_{\mathrm{RF}-}^{\mathrm{ggn}\left(g_{F}\right)}\right|^{2}},
$$

where $\quad E_{F}=A[F(F+1)-I(I+1)-J(J+1)] / 2$, $\omega_{0}=\left|\mu_{B} g_{F} B_{\mathrm{DC}} / \hbar\right|$ the Larmor angular frequency, $\omega_{\mathrm{RF}}^{\operatorname{sgn}\left(g_{F}\right)}$ denotes the rotating frequency of the $\sigma_{\operatorname{sgn}\left(g_{F}\right)}$-polar component of the RF field, defined with respect to the local direction of the static magnetic field ( $z$ axis), and the Rabi coupling:

$$
\Omega_{\mathrm{RF}}^{\mathrm{sgn}\left(g_{F}\right)}=\frac{\mu_{B} g_{F}}{2^{3 / 2} \hbar}\left[-\operatorname{sgn}\left(g_{F}\right) B_{\mathrm{RF}, x} e^{-i \phi_{x}}+i B_{\mathrm{RF}, y} e^{-i \phi_{y}}\right]
$$

with $\operatorname{sgn}\left(g_{F}\right) \in\{+1,-1\}$.

The general definition of the dressed states, $|F, \bar{m}\rangle$ as the Fourier series in Eq. (4) allows us to calculate corrections to the RWA and include the full dependence of the Zeeman shifts with the static magnetic field. This formulation can be extended to the case of polychromatic dressing with $N$ inconmensurable frequencies, using the multidimensional Fourier decomposition of the dressed state:

$$
\langle F, m \mid F, \bar{m}\rangle=\sum_{\vec{n}} U_{m, \bar{m}}^{\vec{n}} e^{i \vec{n} \cdot \vec{\omega} t},
$$


where the $N$-dimensional vectors $\vec{\omega}=\left(\omega_{1}, \omega_{2}, \ldots, \omega_{N}\right)$ and $\vec{n} \in Z^{N}$ [42]. We apply this formalism in Sec. IV to evaluate the dressed energies when applying a bichromatic RF field.

\section{A. Resonant condition in RF-dressed states}

In the experiments described in Sec. III, we perform microwave spectroscopy of the dressed atoms by applying a short and weak MW pulse to the dressed ensemble, followed by the detection of the fraction of atoms remaining in (and transferred to) the initial (the final) hyperfine manifold [23].

To calculate the response of the dressed atom to this pulse, we should express the MW coupling in the dressed basis, using the transformation rule:

$$
\bar{H}_{\mathrm{MW}}=U_{F}^{\dagger}(t) H_{\mathrm{MW}} U_{F}(t),
$$

where $H_{\mathrm{MW}}$ is the atomic coupling to the microwave field expressed in the basis of Zeeman states [see Eq. (1)], and $U_{F}(t)$ is the unitary time-dependent transformation defined in Eq. (4).
The harmonic components of $U_{F}(t)$ combine with the oscillation of the MW field, to produce a resonant condition for transitions between dressed states, $|F, \bar{m}\rangle \leftrightarrow\left|F^{\prime}, \bar{m}^{\prime}\right\rangle$ :

$$
\omega_{\mathrm{MW}}=n \omega_{\mathrm{RF}}+\omega_{\bar{m}^{\prime}, \bar{m}},
$$

with $n \in Z$ and the transition angular frequency:

$$
\omega_{\bar{m}^{\prime}, \bar{m}}=\frac{\bar{E}_{F^{\prime}, \bar{m}^{\prime}}-\bar{E}_{F, \bar{m}}}{\hbar},
$$

where the dressed energy $\bar{E}_{F, \bar{m}}$ is defined in Eq. (3).

The resonant condition between dressed states, Eqs. (9) and (10), is a consequence of couplings of Zeeman substates within each hyperfine manifold, mediated by RF photons. Since the RF-dressed states in the $\bar{m}$ basis are coherent superpositions of $m$ states (Zeeman states), the MW field leads to couplings between the dressed $\bar{m}$ states in different hyperfine manifolds $\left(F, F^{\prime}\right)$, which are resonant at frequencies $\omega_{\mathrm{MW}} / 2 \pi$ that depend on the energy difference between two $\bar{m}$ states. One may also understand this effect as an ac Stark shift of the eigenenergies of the $m$ states mediated by their coupling through RF photons.

Using the RWA and considering near-resonant RF dressing, this resonant condition becomes (see Appendix A):

$$
\begin{aligned}
\omega_{\mathrm{MW}}= & n \omega_{\mathrm{RF}}+\omega_{\mathrm{hfs}}+\frac{g_{F+1}}{\left|g_{F+1}\right|} \bar{m}^{\prime} \sqrt{2}\left|\Omega_{\mathrm{RF}}^{+}\right|-\frac{g_{F}}{\left|g_{F}\right|} \bar{m} \sqrt{2}\left|\Omega_{\mathrm{RF}}^{-}\right|+\left(\frac{g_{F+1}}{\left|g_{F+1}\right|} \frac{\bar{m}^{\prime}}{\left|\Omega_{\mathrm{RF}}^{+}\right|}-\frac{g_{F}}{\left|g_{F}\right|} \frac{\bar{m}}{\left|\Omega_{\mathrm{RF}}^{-}\right|}\right) \frac{\omega_{\mathrm{rf}}^{2}}{2^{3 / 2}} \\
& -\left(\frac{\bar{m}^{\prime} g_{F+1}}{\left|\Omega_{\mathrm{RF}}^{+}\right|}-\frac{\bar{m} g_{F}}{\left|\Omega_{\mathrm{RF}}^{-}\right|}\right) \omega_{\mathrm{RF}} \frac{\mu_{B} B_{\mathrm{DC}}}{\sqrt{2} \hbar}+\frac{1}{2^{3 / 2}}\left[\frac{g_{F+1}}{\left|g_{F+1}\right|} \frac{\bar{m}^{\prime} g_{F+1}^{2}}{\left|\Omega_{\mathrm{RF}}^{+}\right|}-\frac{g_{F}}{\left|g_{F}\right|} \frac{\bar{m} g_{F}^{2}}{\left|\Omega_{\mathrm{RF}}^{-}\right|}\right]\left(\frac{\mu_{B} B_{\mathrm{DC}}}{\hbar}\right)^{2},
\end{aligned}
$$

where $\omega_{\mathrm{hfs}}=(I+1 / 2) A / \hbar$ is the hyperfine splitting.

Experimentally, we scan the MW frequency and determine the resonant condition as a function of the static magnetic field $B_{\mathrm{DC}}$. Thus, we determine the energy difference between dressed states $\hbar \omega_{\bar{m}^{\prime}, \bar{m}}=\left(\bar{E}_{F^{\prime}, \bar{m}^{\prime}}-\bar{E}_{F, \bar{m}}\right)$, observing a quadratic dependence of which we give details below.

\section{SPECTROSCOPY AND PROTECTION OF RF-DRESSED ${ }^{87} \mathbf{R b}$}

In this section, we describe the experimental procedure to achieve decoherence-free pairs of dressed Zeeman sublevels with an ultracold cloud of ${ }^{87} \mathrm{Rb}$ atoms. We focus on the dependence of the microwave resonances with the dressing configuration, which is here controlled by the applied static magnetic field. The main components of our experimental setup are a crossed red-detuned optical trap, a pair of coils that define the magnetic field and our $z$ axis, and three pairs of coils to produce static and RF magnetic fields. For a detailed description we refer to Ref. [23].

We use trapped ultracold atoms so as not to be limited by the free-fall time of the cloud. Instead, our background collisional lifetimes close to two minutes impose no limit on detecting the target coherence times in this work, e.g., $\tau_{\text {coherence }} \approx 100 \mathrm{~ms}$. Furthermore, using an optical dipole trap, instead of a magnetic trap [35], we can generate a stateindependent trapping potential and ensure that the applied RF and DC magnetic fields are nearly homogeneous over the atomic sample. All these features of our setup enable a clean interrogation of the energy difference between RF-dressed states, limited mainly by fluctuations of the dressing field amplitude and polarization.

In our system the magnetic field, taken to be in the $z$ direction, is defined by a homogeneous DC field driven by a pair of Helmholtz coils. However, since we do not implement any magnetic compensation or shielding, both the Earth's magnetic field and fields generated by the equipment nearby, contribute to the total static field. We determine this offset field, $\boldsymbol{B}_{\mathrm{DC}}^{\text {offset }}$, by measuring the resonant frequency of transitions between bare states with a known applied DC field and fitting the Breit-Rabi formula. These measurements are done between the experimental runs that register the dressed spectrum (for details, see Ref. [43]), and we find an offset field with $B_{\mathrm{DC}, z}^{\mathrm{offset}}=(-0.252 \pm 0.014) \mathrm{G}$ and a component in the $x-y$ plane of magnitude $B_{\mathrm{DC}, \perp}^{\mathrm{offset}}=(0.264 \pm 0.012) \mathrm{G}$. This field adds to the one produced by the Helmholtz coils to define total magnetic field $\boldsymbol{B}_{\mathrm{DC}}=\boldsymbol{B}_{\mathrm{DC}}^{H}+\boldsymbol{B}_{\mathrm{DC}}^{\text {offset }}$. We denote its magnitude by $B_{\mathrm{DC}}$.

\section{A. Preparation of the atomic cloud}

Our initial sample is a cold cloud of ${ }^{87} \mathrm{Rb}$ prepared in the bare electronic ground state $|F=1, m=-1\rangle$ [in Fig. 1(a)] via the methods described in detail in Ref. [23]. This cloud is evaporated in a hybrid crossed dipole trap plus a quadrupole (with the gradient $\alpha=10 \mathrm{G} / \mathrm{cm}$ ) until reaching a temperature 


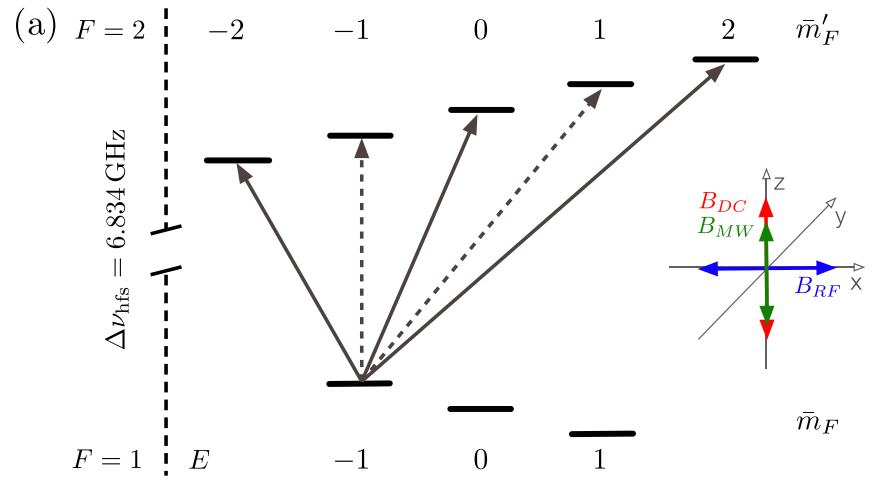

(b)

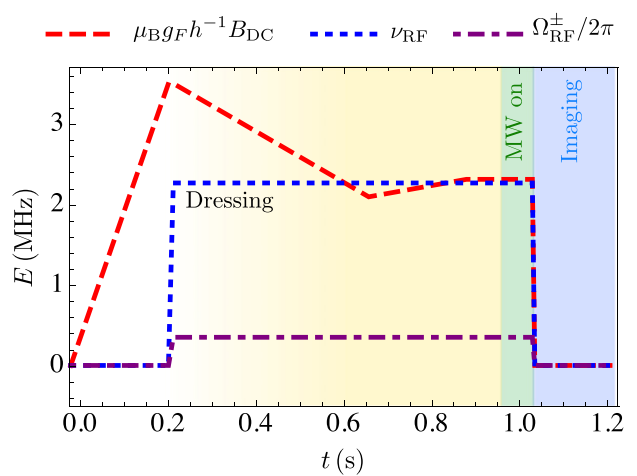

FIG. 1. (a) Schematic of the energy level diagram that indicates available microwave transitions from the initial dressed state $\left|1, \bar{m}_{F}=-1\right\rangle$ state to the upper $F=2$ manifold dressed sublevels. The solid (dashed) arrow lines indicate allowed (nonallowed) transitions for the field configuration shown in the inset. Both the MW and the DC field point in the $z$ direction (purple and green lines, respectively). The RF field is linearly polarized along the $x$ direction. (b) Schematic of the experimental sequence. The red dotted line tracks the DC field, the dashed blue line tracks the RF frequency, and the purple dashed line tracks the RF amplitude, represented by the Rabi frequency. All curves are shown in frequency units. The grayed-out areas represent the MW (green) and imaging (blue) pulses, respectively. The shaded yellow are represents the adiabatic dressing of the atoms. The ramp times of $B_{\mathrm{DC}}, \nu_{\mathrm{RF}}=\omega_{\mathrm{RF}} /(2 \pi)$, and $\Omega_{\mathrm{RF}}$ are those described in Sec. III. The extent of the "MW on" and "Imaging" grayed-out areas does not represent their real duration.

of $100 \mathrm{nK}$ with a typical population of $n=2 \times 10^{5}$ atoms in the bare state $|1,-1\rangle$. A bias field in the vertical direction $B_{\mathrm{DC}}^{H} \approx 1.2 \mathrm{G}$, produced by a pair of Helmholtz coils, is present at this point, too. The dipole trap has been tuned at $\alpha=2 \mathrm{G} / \mathrm{cm}$ so that the trapping frequencies are $\omega_{\rho} / 2 \pi \approx$ $180 \mathrm{~Hz}$ and $\omega_{\text {axial }} / 2 \pi \approx 30 \mathrm{~Hz}$. We subsequently switch off the current to the quadrupole coils, leaving a residual magnetic quadrupole field with a maximal measured field-gradient of $\alpha<0.05 \mathrm{G} / \mathrm{cm}$.

In the next step, the atoms are dressed through the procedure sketched in Fig. 1(b): The current in the pair of Helmholtz coils is ramped up to increase the applied static field from $\boldsymbol{B}_{\mathrm{DC}}^{H}=0 \hat{z} \mathrm{G}$ to $\boldsymbol{B}_{\mathrm{DC}}^{H}=5 \hat{z} \mathrm{G}$ in $\Delta t=200 \mathrm{~ms}$. At that point, a linearly polarized RF field in the $x$ direction with angular frequency $\omega_{\mathrm{RF}} / 2 \pi \approx 2.3 \mathrm{MHz}$ and Rabi coupling $\sqrt{2}\left|\Omega_{\mathrm{RF}}^{ \pm}\right| / 2 \pi \approx 350 \mathrm{kHz}$ is switched on, followed by an adiabatic linear ramp (of duration $\Delta t=0.475 \mathrm{~s}$ ) from
$\boldsymbol{B}_{\mathrm{DC}}^{H}=5 \hat{z} \mathrm{G}$ to $\boldsymbol{B}_{\mathrm{DC}}^{H}=3 \hat{z} \mathrm{G}$. After this, the magnitude of the Helmholtz field, $B_{\mathrm{DC}}^{H}$, is increased to a final value so that the total field $B_{\mathrm{DC}}$ is within the range [3.1,3.3] G. Finally, we probe the RF-dressed atom with a MW pulse and take quasisimultaneous independent absorption images of the $F=1$ and $F=2$ manifolds, shortly after switching off the dipole trap that holds the cloud [43]. We will refer to the number of atoms measured in $F=2(F=1)$ as $n_{2}\left(n_{1}\right)$, and the fraction of atoms in $F=2$ as $f_{2}=n_{2} /\left(n_{1}+n_{2}\right)$.

\section{B. Protected transitions with ultracold RF-dressed atoms}

After preparing the atomic cloud in the dressed state $|1, \bar{m}=-1\rangle$, we fix the dressing frequency $\omega_{\mathrm{RF}}^{ \pm} / 2 \pi=$ $2.263410 \mathrm{MHz}$ and Rabi couplings $\sqrt{2}\left|\Omega_{\mathrm{RF}}^{ \pm}\right| / 2 \pi \approx 350 \mathrm{kHz}$. Then we determine the resonant frequency of three transitions in the vicinity of the zero field hyperfine splitting [23] as functions of the applied static field $B_{\mathrm{DC}}^{H}$. For each value of the total static magnetic field, $B_{\mathrm{DC}}$, we extract the resonant transition frequency after fitting Lorentzian curves to measurements of the transferred atomic-state population following a short microwave pulse and scanning the microwave frequency. The transitions investigated have the final states $|2, \bar{m}=1\rangle$ (at $\left.\omega_{\mathrm{MW}} \approx \omega_{\mathrm{hfs}}\right),|2, \bar{m}=2\rangle\left(\right.$ at $\left.\omega_{\mathrm{MW}} \approx \omega_{\mathrm{hfs}}+\sqrt{2}\left|\Omega_{\mathrm{RF}}^{+}\right|\right)$, and $|2,0\rangle$ (at $\left.\omega_{\mathrm{MW}} \approx \omega_{\mathrm{hfs}}-\sqrt{2}\left|\Omega_{\mathrm{RF}}^{+}\right|\right)$, and the corresponding resonant frequencies can be estimated from Eq. (9). Results of these measurements are shown in Fig. 2. When sampling the total field $B_{\mathrm{DC}}$ near the resonant condition of the RF field with the Larmor frequency, we observed a linear dependence of the resonant transition frequency to the state $|2, \bar{m}=1\rangle$, and a quadratic dependence for the transitions to $|2, \bar{m}=2\rangle$ and $|2, \bar{m}=0\rangle$, which is in qualitative agreement with Eqs. (5)-(9) after taking into account the difference between Landé factors of the two hyperfine manifolds. Even though we represented the transition to the $|2,1\rangle$ state as nonallowed in Fig. 1, this occurs only in the idealized case where all fields are aligned as the inset of this figure indicates, see Ref. [23].

We also observe that the transition lines from the dressed state $|1, \bar{m}=-1\rangle$ to $|2, \bar{m}=0\rangle$ and $|2, \bar{m}=2\rangle$ narrow down to a linewidth of only $\Delta v \approx 110 \mathrm{~Hz}$ (for a MW pulse duration of $\Delta t=10 \mathrm{~ms}$ ) when the static field is adjusted to the extrema of the quadratic line shifts, even without using active stabilization of $B_{\mathrm{DC}}$ or magnetic shielding.

In Fig. 3 we show the line shape of the transition $\mid 1, \bar{m}=$ $-1\rangle \rightarrow|2, \bar{m}=2\rangle$ for three particular total fields $B_{\mathrm{DC}}=$ 3.197 G, 3.216 G, and 3.247 G. This figure shows the fraction of atoms transferred to the $F=2$ manifold as a function of the applied microwave frequency, in the vicinity of the hyperfine splitting frequency, $\omega_{\mathrm{hfs}}$. The most striking feature of these measurements is the narrowing of the line shape as the static field approaches the turning point of the quadratic line shift in Fig. 2(a), where the transition becomes protected against fluctuations of the DC field.

Our data in Fig. 2(b) shows that the transition to $\mid 2, \bar{m}=$ 1) presents a remarkably different behavior compared to the other two. In this case, the resonant frequency presents a linear dependence with the static field in the vicinity of the single MW photon transition [i.e., with $n=0$ in Eq. (9)], preventing us from finding a condition to null the DC field sensitivity of 


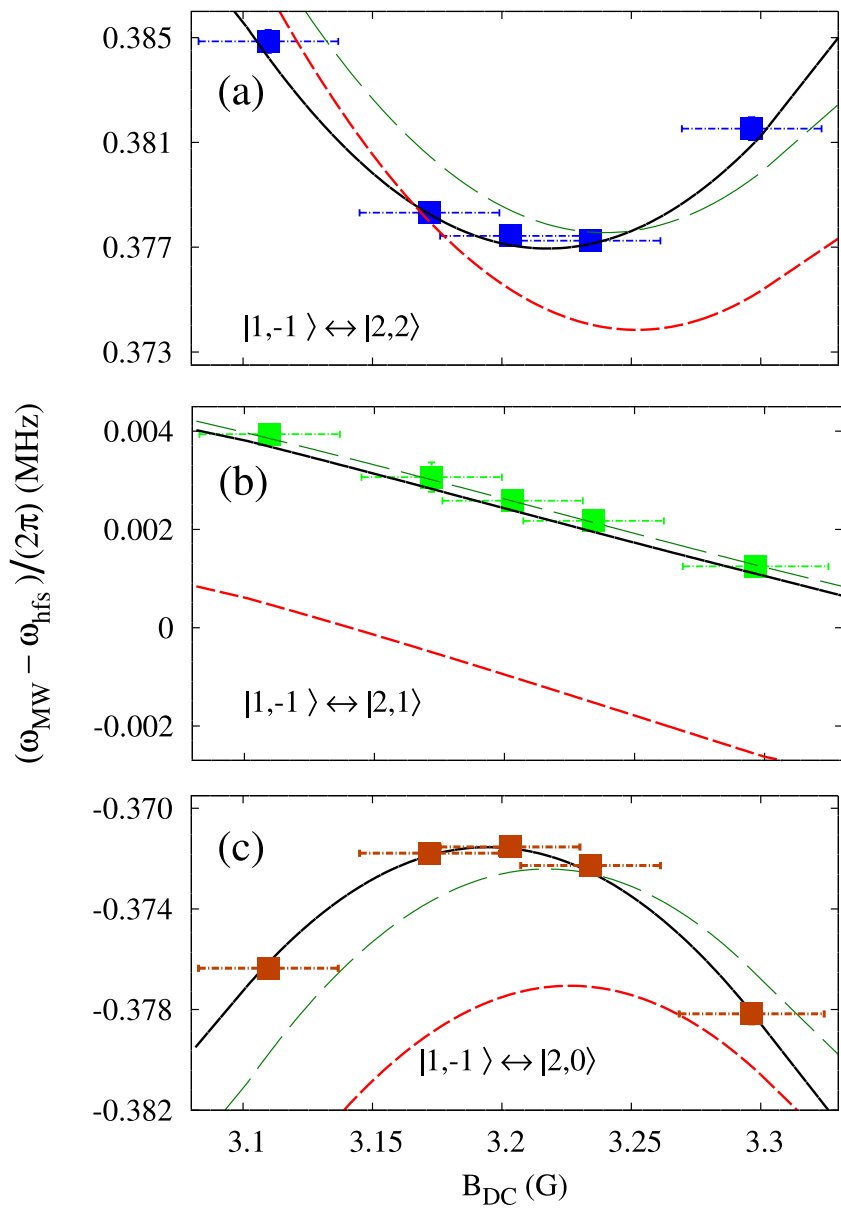

FIG. 2. Detuning of the resonant frequencies of the transitions between the dressed states (a) $|1,-1\rangle \rightarrow|2,2\rangle$, (b) $|1,-1\rangle \rightarrow$ $|2,1\rangle$, and (c) $|1,-1\rangle \rightarrow|2,0\rangle$ as functions of the applied static magnetic field. The symbols correspond to experimental values obtained from spectral signals. The solid lines are fits to our model, in Sec. II, which take into account nonlinear Zeeman shifts and beyond RWA effects. The long-dashed (short-dashed) lines are fits to a model considering the RWA and nonlinear (linear) Zeeman shifts. The amplitude of the RF field is the only free parameter of our models since we measure the total static magnetic field. For the full model (longdashed) we obtained $B_{\mathrm{RF}}^{+}=(1.07 \pm 0.09) \mathrm{G}$, while for for the solid lined $B_{\mathrm{RF}}^{+}=(1.08 \pm 0.09) \mathrm{G}$, with $B_{\mathrm{RF}}^{\mathrm{sgn}\left(g_{F}\right)}=\sqrt{2}\left|\Omega_{\mathrm{RF}}^{\mathrm{sgm}\left(g_{F}\right)} /\left(\mu_{B} g_{F}\right)\right|$. The short-dashed line is evaluated using $B_{\mathrm{RF}}^{+}=1.075 \mathrm{G}$ and the measured DC magnetic field.

this transition. However, as shown in Fig. 4(a), the resonant frequency displays a turning point when the magnitude of the DC field enables a two-photon transition, which corresponds to the resonant condition with $n=1$ in Eq. (9) [23]. Under this condition, we consistently measured coherence times $>$ $50 \mathrm{~ms}$ in a Ramsey type experiment in the crossed-dipole trap, which quickly shortens when setting the DC field away from the turning point [44].

Figures 4(a) and 4(b) illustrate the behavior of the transition $|1, \bar{m}=-1\rangle \rightarrow|2, \bar{m}=1\rangle$ far from the resonant point of the RF frequency with the Larmor frequency and with atoms trapped in a crossed-dipole potential, as before. Figure 4(a) shows the dependence with the static field of the detuning of the resonant frequency defined as $\delta_{\mathrm{MW}}=\left(\omega_{\mathrm{MW}}-\omega_{\mathrm{hfs}}-\right.$

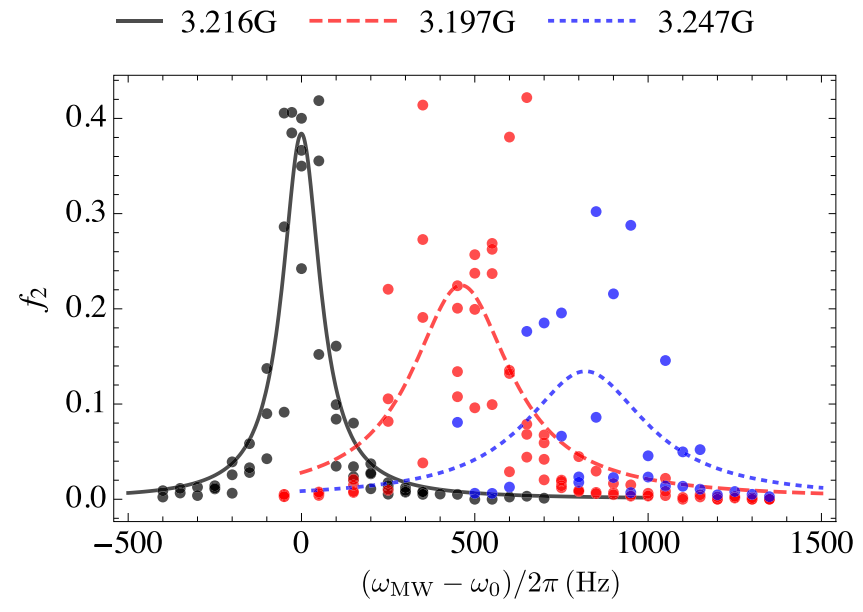

FIG. 3. Experimental demonstration of line narrowing in the dressed RF system. The measurements (dots) of the transition $|1, \bar{m}=-1\rangle \rightarrow|2, \bar{m}=2\rangle$ for three different total fields $B_{\mathrm{DC}}$ : $3.195 \mathrm{G}$ (red), $3.247 \mathrm{G}$ (blue), and 3.216 G (black). The latter case is chosen to be optimal at $\omega_{0} / 2 \pi=2.25059 \mathrm{MHz}$, which defines zero on the horizontal axis and corresponds to the minimum of the parabola in Fig. 2(a). In all cases the MW pulse duration is $\Delta t=10$ ms. The horizontal axis is the detuning $\Delta \omega_{0}=\omega_{\mathrm{MW}}-\omega_{0}$ with respect to the minimum of the parabola, which we have labeled $\omega_{0}$. The vertical axis shows the fraction of measured population in $F=2$, that we defined as $f_{2}$ in Sec. III A. The lines are fits to Lorentzian curves for the three total magnetic fields: $B_{\mathrm{DC}}=3.195 \mathrm{G}$ (dashed red), $B_{\mathrm{DC}}=3.247 \mathrm{G}$ (dotted blue), and $B_{\mathrm{DC}}=3.216 \mathrm{G}$ (solid black). One can see that the randomness of the transition increases as one interrogates further away from the condition that minimizes the parabola in Fig. 2(a). This is due to the higher sensitivity to DC magnetic field fluctuations. The fitted Lorentzian linewidths are as follows: $\Delta v=(132 \pm 13) \mathrm{Hz}, \Delta v=(347 \pm 54) \mathrm{Hz}$, and $\Delta v=$ $(427 \pm 189) \mathrm{Hz}$ for the black, red, and blue curves, respectively.

$\left.\omega_{\mathrm{RF}}\right) / 2 \pi$, where $\omega_{\mathrm{hfs}}$ is the hyperfine splitting frequency and $\omega_{\mathrm{RF}} / 2 \pi=2.27 \mathrm{MHz}$. The black dots correspond to the center of the Lorentzian fits in Fig. 4, and the black dotted line is included as a guide to the eye. We also observe the intermittent appearance of a second, weaker peak (see Appendix B) marked by gray squares and a dashed line. This feature comes from transitions from the bare state $|1,1\rangle$, which becomes populated by nonadiabatic effects when sweeping the applied DC field [45]. In the same panel, the vertical dashed line (blue) indicates the turning point of the curve, where $\partial \delta_{\mathrm{MW}} / \partial B_{\mathrm{DC}}=0$, and the vertical dotted line (red) points to a static field with a comparatively large gradient of $\delta_{\mathrm{MW}}$.

Figure 4(b) shows two sets of data in a Ramsey-type experiment. The blue set of data is taken at the turning point of the inverted parabola in Fig. 4(a), corresponding to $B_{\mathrm{DC}}=$ $2.56 \mathrm{G}$. The red set of data is taken with $B_{\mathrm{DC}}=2.87 \mathrm{G}$, i.e., where the vertical red dotted line indicates in (a). For these experiments, we detune the driving MW pulse from each transition by $\delta v_{R}=v_{\mathrm{MW}}-v_{0}$, where $v_{0}$ is the transition frequency and $v_{\mathrm{MW}}$ is the MW frequency, and then drive the transition off-resonantly with two MW pulses separated by $\tau_{\text {Ramsey }}$. We then scan $\tau_{\text {Ramsey }}$ and observe the free temporal evolution of the population in the upper dressed state $|2,1\rangle$. 

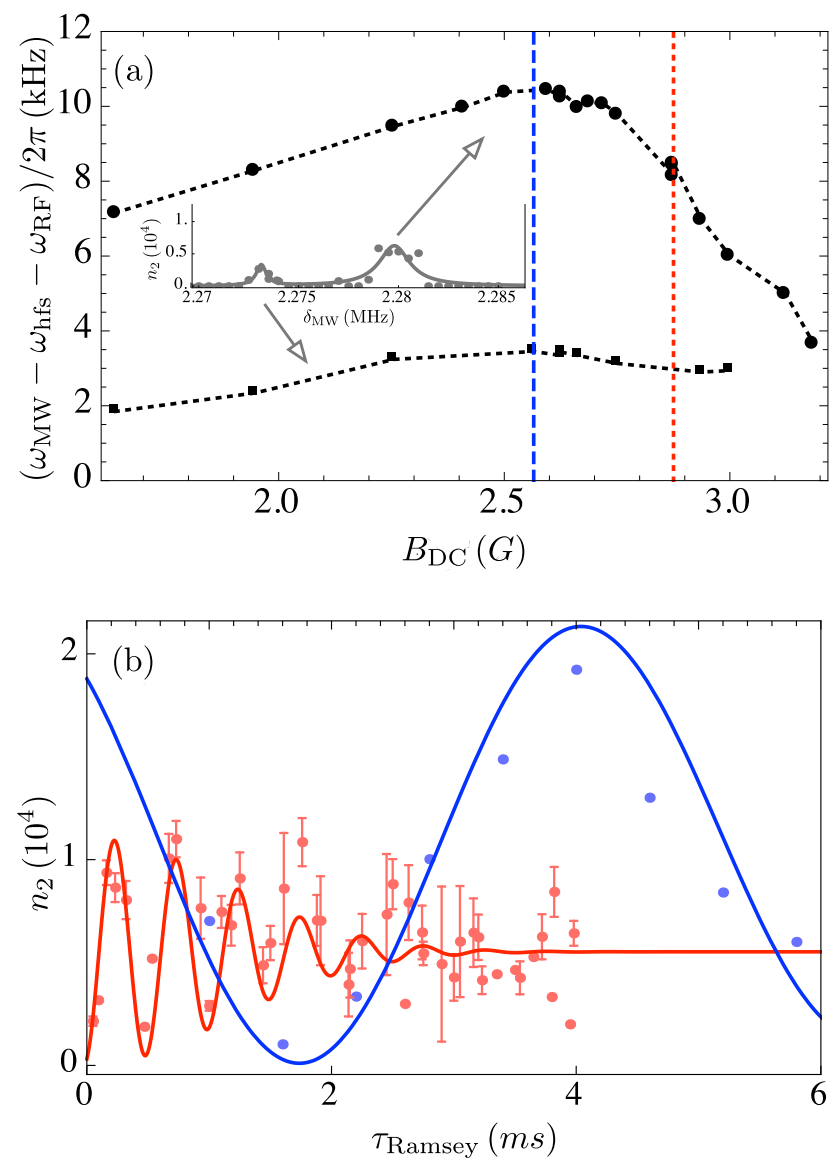

FIG. 4. Line shift and Ramsey fringes observed in ${ }^{87} \mathrm{Rb}$ dressed with the Rabi frequency $\Omega_{\mathrm{RF}} / 2 \pi=300 \mathrm{KHz}$ and the RF frequency $\omega_{\mathrm{RF}} / 2 \pi=2.27 \mathrm{MHz}$, as functions of the static magnetic field. (a) The line shift for the $|1, \bar{m}=-1\rangle \rightarrow|2, \bar{m}=1\rangle$ transition. We observe a second weaker peak in the range of the static field explored (see Appendix B). Inset: A typical spectral signal with two Lorentzian peaks matched to data in the main panel. (b) Ramsey fringes of the atomic population of the upper hyperfine manifold, $n_{F=2}$, when $B_{\mathrm{DC}}=2.87 \mathrm{G}$ (red line) and $B_{\mathrm{DC}}=2.56 \mathrm{G}$ (blue line). The vertical dashed and dotted lines in (a) indicate the values of the magnetic fields used to obtain the Ramsey fringes in (b). Also in (b), the red dots with error bars are rms values and standard deviations, respectively, obtained from five repetitions per point. Red and blue dots without error bars are single measurements of the total number of atoms in the $F=2$ manifold, $n_{2}$. To highlight the contrast between the noise-sensitive (red dots) and the protected (blue data) dressing configurations, we only show the first $65 \mathrm{~ms}$ of a longer experimental run (not shown). Considering Ramsey fringes modelled with exponential decay, the protected configuration (blue dots) displays a dephasing time of $\tau=17 \mathrm{~ms}$ while the noise-sensitive case shows a dephasing time of $\tau=1.65 \mathrm{~ms}$.

In both cases we fit the atom number to $f_{2}=\left(n_{2} / 2\right)[1+$ $\left.e^{-\frac{t^{2} \sigma^{2}}{2}} \cos \left(2 \pi t \delta v_{R}\right)\right]$, where $\sigma$ is the standard deviation of a Gaussian-shaped noise profile associated with the two-level system defined by the states $|1, \bar{m}=-1\rangle$ and $|2, \bar{m}=1\rangle$. The fit to the blue set of data gives $\delta v_{R}=216 \pm 2 \mathrm{~Hz}$ and $\sigma=79 \pm 8 \mathrm{~Hz}$, whereas the fit to the red set of data gives $\delta v_{R}=1972 \pm 35 \mathrm{~Hz}$ and $\sigma=883 \pm 112 \mathrm{~Hz}$. The MW pulse detuning in the red (blue) set of data was chosen to be $\delta v_{R}=$
$2000 \mathrm{~Hz}\left(\delta v_{R}=200 \mathrm{~Hz}\right)$, which agrees with the fit estimate. The difference in dephasing rates of the two Ramsey oscillations is a result of the distinct DC-field noise sensitivity of the dressed transitions (quantified by the gradient $\partial \delta_{\mathrm{MW}} / \partial B_{\mathrm{DC}, z}$ ) at the chosen static fields on Fig. 4(a). The different amplitude of the oscillations is due to the relative shift of the driving field detuning with respect to resonant driving, which in the case of the red curve it is as much as $2 \mathrm{kHz}$.

In both cases, the fluctuations of the RF field still shift the transitions and contribute to the broadening of the line shape. For instance, in the transition $|1, \bar{m}=-1\rangle \rightarrow|2, \bar{m}=1\rangle$, we measured a line shift of $7 \mathrm{~Hz}$ per $\mathrm{kHz}$ of Rabi frequency caused by the RF amplitude noise. It is evident in any case that the DC-noise fluctuations result in a much larger dispersion of the measurements as the lines are further detuned from the extreme of the parabola-shaped line shift. This produces a better Lorentzian fit for the black data set in Fig. 3 and worse fits for the blue and red data sets. Both Fig. 3 and Fig. 4(b) show that transitions between Zeeman substates can be narrowed down in the RF-dressed regime by choosing field parameters where the sensitivity to DC fields of the differential energy shifts become minimal, although the optimal configuration depends on the pair of states involved.

\section{SIMULTANEOUS DC PROTECTION OF MULTIPLE DRESSED TRANSITIONS}

\section{A. Principles of extended protection}

The protection against DC-field fluctuations demonstrated in the previous section (Sec. III) occurs at different DC fields for different transitions. In particular, we observed how the dressing configuration can be adjusted to reduce strongly the sensitivity of the dressed transitions $|F=1, \bar{m}=-1\rangle \rightarrow$ $|F=2, \bar{m}=2\rangle$ (see Fig. 3) and $|F=1, \bar{m}=-1\rangle \rightarrow \mid F=$ $2, \bar{m}=1\rangle$ (see Fig. 4). This configuration is possible thanks to the nearly identical dependence of the dressed energies involved with the applied static field. These dependencies result in a condition where, to first order, variations of the static field do not affect the transition angular frequency:

$$
\left.\frac{\partial \omega_{\bar{m}^{\prime}, \bar{m}}}{\partial B_{\mathrm{DC}}}\right|_{B_{\mathrm{DC}}^{0}}=0 .
$$

at a particular value of the static field, $B_{\mathrm{DC}}^{0}$. In this section, we propose to use a combination of two RF fields to manipulate the dressed energy of the hyperfine manifolds $F=1$ and $F=2$ independently of each other, and investigate how this scheme can be used to reduce the DC-magnetic field sensitivity of several transitions at the same value of the static field.

Considering only the RWA, the upper and lower hyperfine manifolds are dressed independently by different circular component of the RF dressing field. Also, assuming that a static magnetic field of amplitude $B_{\mathrm{DC}}^{0}$ produces a linear Zeeman shift, we find that all transitions between dressed states become protected when the frequency of each polar component is set according to:

$$
\begin{gathered}
\hbar \omega_{\mathrm{RF}}^{\operatorname{sgn}\left(g_{F+1}\right)}=\mu_{B}\left|g_{F+1}\right| B_{\mathrm{DC}}^{0}, \\
\hbar \omega_{\mathrm{RF}}^{\mathrm{sgn}\left(g_{F}\right)}=\mu_{B}\left|g_{F}\right| B_{\mathrm{DC}}^{0}
\end{gathered}
$$


(a)
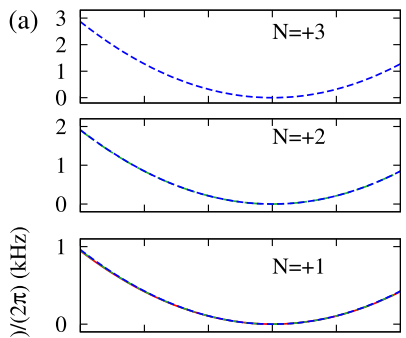

$+ \stackrel { - } { \sim } 1 \longdiv { \mathrm { N } = 0 }$

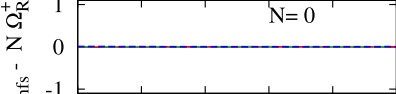

उำ
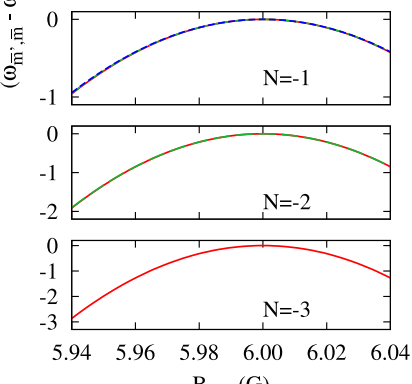

$\mathrm{B}_{\mathrm{DC}}(\mathrm{G})$

FIG. 5. Resonant frequencies of all hyperfine transitions in RF dressed ${ }^{87} \mathrm{Rb}$, as functions of the static magnetic field. An offset is applied to each resonant frequency to define a simple vertical scale with a similar range for all cases. In all panels, the dressing field configuration is given by Eqs. (13)-(15), with $B_{\mathrm{DC}}^{0}=6.0 \mathrm{G}$, $\bar{m}=-\bar{m}^{\prime}=1$, and the $B_{\mathrm{RF}}^{+}=0.12 \mathrm{G}$. The dressed energies are calculated using (a) the RWA and (b) the dressing scheme in Eq. (7) with two frequencies. Solid, dashed, and short-dashed lines correspond to transitions with the initial states $|F=1, \bar{m}\rangle=|1,-1\rangle,|1,0\rangle,|1,1\rangle$, respectively. The labels indicate the final states as $\mid F=2, \bar{m}^{\prime}=$ $N-\bar{m}\rangle$. In panel (b), the nonlinear Zeeman shift and beyond RWA effects break the degeneracy of the transitions observed in panel (a).

i.e., the resonant dressing of each hyperfine manifold ensures that Eq. (12) is satisfied exactly for all transitions.

Furthermore, the RWA also tells us that when the polar components of the dressing field satisfy the condition:

$$
\frac{B_{\mathrm{RF}}^{\mathrm{sgn}\left(g_{F+1}\right)}}{B_{\mathrm{RF}}^{\mathrm{sgn}\left(g_{F}\right)}}=\frac{\bar{m}}{\bar{m}^{\prime}} \frac{g_{F}}{g_{F+1}},
$$

with $B_{\mathrm{RF}}^{\mathrm{sgn}\left(g_{F}\right)}=\sqrt{2}\left|\Omega_{\mathrm{RF}}^{\mathrm{sgn}( \pm)} /\left(\mu_{B} g_{F}\right)\right|$, all transitions between states with the same sign of the magnetic moment are also stable to second order:

$$
\left.\frac{\partial^{2} \omega_{\bar{m}^{\prime}, \bar{m}}}{\partial B_{\mathrm{DC}}^{2}}\right|_{B_{\mathrm{DC}}^{0}}=0 .
$$

The mechanism behind the reduction of the sensitivity of multiple transitions is shown in Fig. 5, where we plot the detuning of the resonant frequencies of all 15 possible transitions, $\delta_{\bar{m}^{\prime}, \bar{m}}=\left(\omega_{\bar{m}^{\prime}, \bar{m}}-\omega_{\mathrm{hfs}}\right) / 2 \pi$, as functions of the DC field. To see the effect, in Fig. 5(a), we use $B_{\mathrm{DC}}^{0}=6.0 \mathrm{G}$ and $B_{\mathrm{RF}}^{+}=0.12 \mathrm{G}$ along with Eqs. (13)-(15) for the frequencies and amplitudes of the circular components of the RF field. In this case, the resonant frequency of all transitions, $\delta_{\bar{m}^{\prime}, \bar{m}}$, display one equilibrium point at our chosen value $B_{\mathrm{DC}}^{0}=6.0 \mathrm{G}$ (i.e., $\partial \delta_{\bar{m}^{\prime}, \bar{m}} /\left.\partial B_{\mathrm{DC}}\right|_{B_{\mathrm{DC}}^{0}}=0, \forall\left\{\bar{m}^{\prime}, \bar{m}\right\}$ ), which corresponds to ensuring first-order (i.e., linear) protection against noise in the static field. In Fig. 5(b), we plot the dressed energy calculated taking into account nonlinear Zeeman shifts and beyond RWA effect, using the method outlined in Sec. II. In contrast to the previous case, now the equilibrium points of $\delta_{\bar{m}^{\prime}, \bar{m}}$ occur at different values of the static field, significantly distinct from $B_{\mathrm{DC}}$. We conclude that, in general, the conditions Eqs. (13)-(15), do not protect the states against fluctuations of the DC field at the applied field $B_{\mathrm{DC}}$ because of nonlinear effects breaking the regularity of the energy spectrum assumed by our RWA model. Note, however, that with these conditions all resonant conditions experience a similar, but not identical, shift toward $B_{\mathrm{DC}} \approx 5.93 \mathrm{G}$.

\section{B. Sensitivity of the bichromatic driven atom to DC amplitude noise}

We quantify the average DC sensitivity of the dressed atom using the rms of the first derivative of the 15 microwave transition frequencies with respect to the static field:

$$
\alpha_{\mathrm{DC}}=\frac{1}{2 \pi} \sqrt{\frac{1}{15} \sum_{\bar{m}=-1}^{1} \sum_{\bar{m}^{\prime}=-2}^{2}\left(\frac{\partial \omega_{\bar{m}^{\prime}, \bar{m}}}{\partial B_{\mathrm{DC}}}\right)^{2}} .
$$

The dressed transition frequencies in Fig. 5(b) indicate that the nonlinear Zeeman shifts and beyond RWA effects frustrate the exact first-order stability expected when applying the RWA when we obtain $\alpha_{\mathrm{DC}}=0$.

Setting the dressing frequencies and amplitudes according to Eqs. (13) and (14), we evaluated numerically $\alpha_{\mathrm{DC}}$ for a typical range of experimentally relevant parameters. Our numerical results, in Fig. 6, show that, when using the conditions indicated by the RWA and for sufficiently strong RF fields (e.g., $B_{\mathrm{RF}}^{+}>0.08 B_{\mathrm{DC}}$ ), the atomic sensitivity $\alpha_{\mathrm{DC}}$ depends linearly on the amplitude of the RF field and is independent of the applied static field. For weaker RF fields, $\alpha_{\mathrm{DC}}$ depends on the static field since both beyond-RWA and nonlinear Zeeman effects become comparable.

The residual sensitivity observed in Fig. 6 can be reduced by adjusting the dressing frequencies to bring them back into resonance after taking into account energy shifts induced by the driving. Such energy shifts occur because, while each circular component of the RF field dresses one hyperfine manifold, they also cause off-resonant perturbations of the other one [28]. Using a second-order perturbative expansion of the RWA dressed energy, these energy shifts translate into a correction of the resonant condition given by (see Appendix C):

$$
\hbar \Delta \omega_{\mathrm{RF}}^{\ell_{F}}=\frac{1}{2} \frac{\left|\mu_{B} g_{F} B_{\mathrm{RF}}^{-\ell_{F}}\right|^{2}}{\mu_{B}\left|g_{F}\right| B_{\mathrm{DC}}+\hbar \omega_{\mathrm{RF}}^{-\ell_{F}}},
$$

with $\ell_{F}=\operatorname{sgn}\left(g_{F}\right)$ and $F=I \pm 1 / 2$.

With these arguments as a guide for our calculations, we numerically optimize the combination of frequencies that minimize $\alpha_{\mathrm{DC}}$, using field amplitudes at the ratio given by Eq. (15). The fractional frequency shift with respect to the conditions given by the RWA is shown in Fig. 7, which is in qualitative agreement with Eqs. (18). Note that, although the relative frequency shift is of order $\sim 10^{-3}$, this translates 


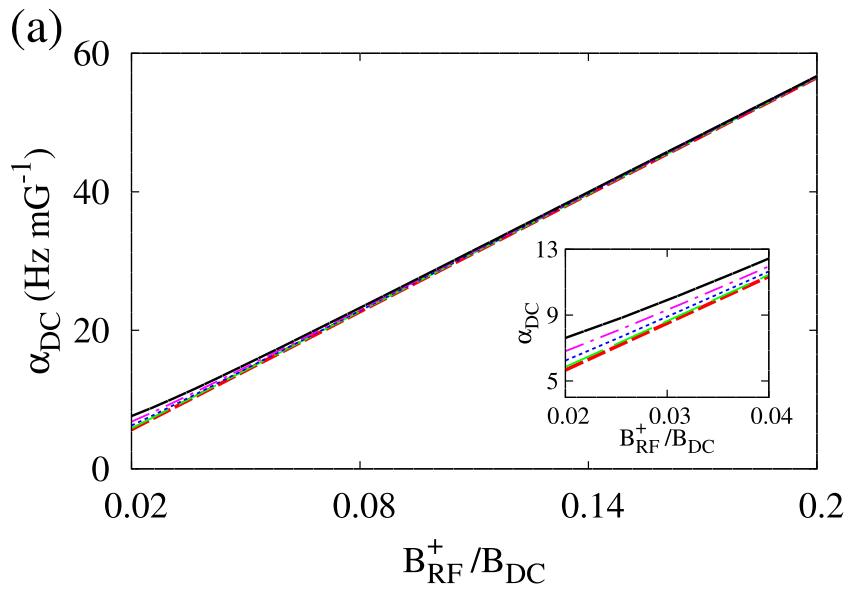

(b)

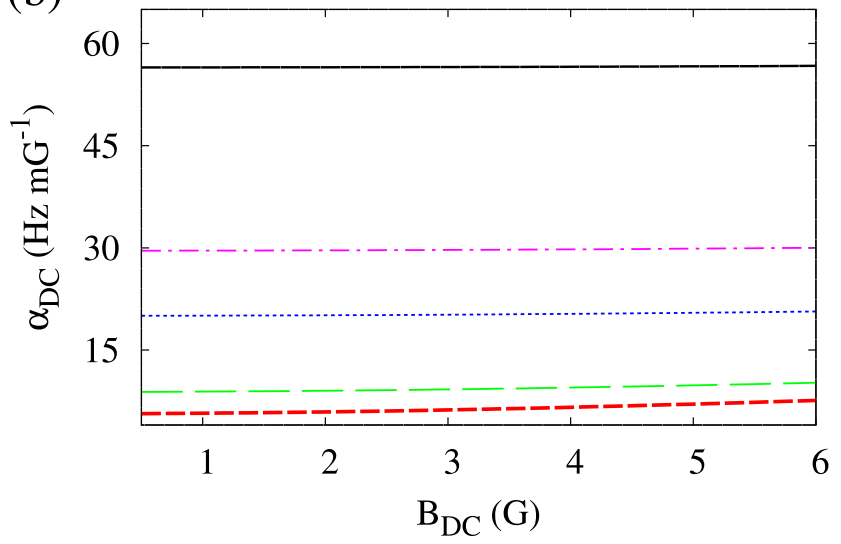

FIG. 6. Average sensitivity $\alpha_{\mathrm{DC}}$ of the dressed atomic transitions with respect to the static field [Eq. (17)], calculated using the field configuration given by the RWA Eqs. (13)-(15). Panel (a) shows $\alpha_{\mathrm{DC}}$ as a function of the ratio $B_{\mathrm{RF}}^{+} / B_{\mathrm{DC}}$ for the DC fields $0.5 \mathrm{G}$ (short-dashed red), $1.70 \mathrm{G}$ (dashed green), $3.08 \mathrm{G}$ (dotted blue), 4.28 G (dot-dashed cyan), and 6.0 G (solid black). The inset shows the expanded region of weak fields. Panel (b) shows $\alpha_{\mathrm{DC}}$ as a function of the static field, $B_{\mathrm{DC}}$, for RF fields of amplitude $0.5 \mathrm{G}$ (short-dashed red), $1.70 \mathrm{G}$ (dashed green), $3.08 \mathrm{G}$ (dotted blue), $4.28 \mathrm{G}$ (dasheddot cyan), and $6.0 \mathrm{G}$ (solid black).

into an important correction in absolute terms, typically corresponding to a shift of a few $\mathrm{kHz}$ of the $\mathrm{RF}$ frequency.

When using the optimized frequencies, $\alpha_{\mathrm{DC}}$ is reduced by one order of magnitude and becomes weakly dependent on the amplitude of the RF field (since we compensate for their main contribution to the energy shifts). It also becomes linearly dependent on the static field, as shown in Fig. 8 [46].

The effect of using corrected RF frequencies is presented in Fig. 9, where we plot the 15 dressed transition frequencies as functions of the static magnetic field. Note that in this case the extrema of all curves $\omega_{\bar{m}^{\prime}, \bar{m}}$ return to the vicinity of $B_{\mathrm{DC}}=$ 6.0G [compare with Figs. 5(a) and 5(b)].

\section{Fractional frequency fluctuations of a bichromatic dressed atom}

Finally, we illustrate quantitatively the improved stability enabled by the optimized bichromatic RF dressing using the

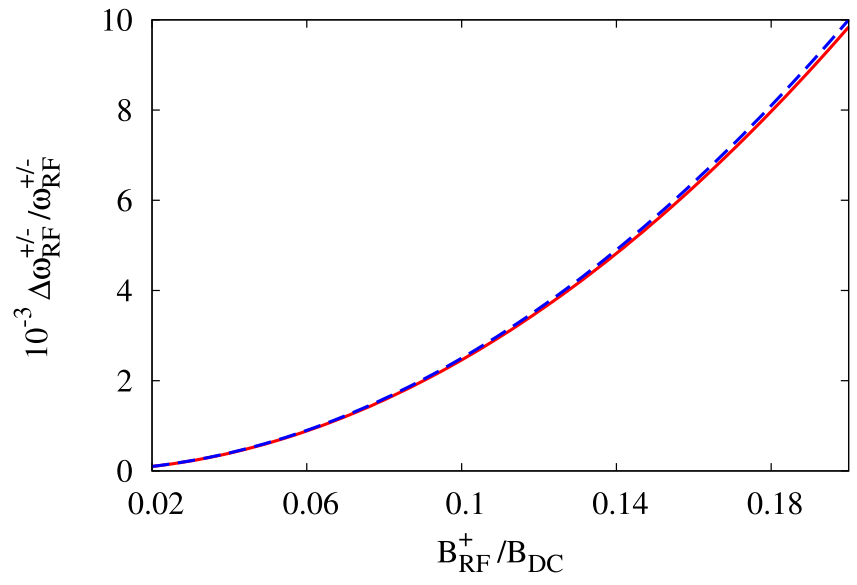

FIG. 7. Fractional correction of the frequencies required to reduce the average atomic linear sensitivity with respect to the static field: Frequency of the $\sigma_{\operatorname{sgn}\left(g_{F+1}\right)}$ (dashed) and $\sigma_{\operatorname{sgn}\left(g_{F}\right)}$ (solid) polar components of the dressing fields. This shift is independent of the applied static field.

fractional frequency fluctuation due to noise in the DC field, defined by:

$$
\sigma_{\bar{m}^{\prime}, \bar{m}}=\frac{1}{\omega_{\bar{m}^{\prime}, \bar{m}}} \frac{\partial \omega_{\bar{m}^{\prime}, \bar{m}}}{\partial B_{\mathrm{DC}}} \times \Delta B_{\mathrm{DC}} .
$$

For concreteness, in Table I, we consider all transitions between the state $|1, \bar{m}=-1\rangle$ to the five states of the upper hyperfine manifold, and perform this calculation for four comparable field configurations: (A) bare atom, (B) monochromatic linearly polarized RF dressing with frequency $\omega_{\mathrm{RF}}=\mu_{B}\left|g_{2}-g_{1}\right| B_{\mathrm{DC}} /(2 \hbar)$ and $B_{\mathrm{RF}, x}=0.2 B_{\mathrm{DC}},(\mathrm{C})$ bichromatic dressing with the dressing field given by the RWA, Eqs. (13)-(15) and using $B_{\mathrm{RF}}^{+}=0.1 B_{\mathrm{DC}}$, and (D) optimized bichromatic driving with $B_{\mathrm{RF}}^{+}=0.1 B_{\mathrm{DC}}$. In all these cases, we consider DC fluctuations of amplitude $\Delta B_{\mathrm{DC}}=0.1 \mathrm{mG}$.

The best stability is obtained for the transition $\mid 1, m=$ $-1\rangle \rightarrow|2, m=1\rangle$ of the bare atom (A) at the magic field $B_{\mathrm{DC}}=3.22 \mathrm{G}$. However, all dressing configurations considered provide a global improvement over the fluctuations of two orders of magnitude for all transitions. In particular, the optimized bichromatic driving configuration (D) defines all transitions with the same level of protection.

Noise in the amplitude of the dressing field contributes to the linewidth of the spectral lines of transitions between dressed states. Temporal variations of each polar component of the RF field affect the transition frequencies by modifying the dressed energy of the manifold it dresses and induce corrections to the perturbative energy shift of the other hyperfine manifold. We use the rms variation of the transition frequencies $\left[\omega_{\bar{m}, \bar{m}^{\prime}} /(2 \pi)\right]$ with respect to each polar component to quantify these effects in Appendix C. The average first-order sensitivity of the transition frequencies are of order $\approx 10^{2} \mathrm{~Hz} \mathrm{mG}^{-1}$; much larger than the residual DC sensitivity $\alpha_{\mathrm{DC}} \lesssim 10 \mathrm{~Hz} \mathrm{mG}^{-1}$ achieved by tuning the frequencies of each polar component of the RF field. Thus, the improved stability of the bichromatic dressing scheme becomes useful when there is equally good stability of the $\mathrm{RF}$ source. The bichromatic dressing configuration has been 

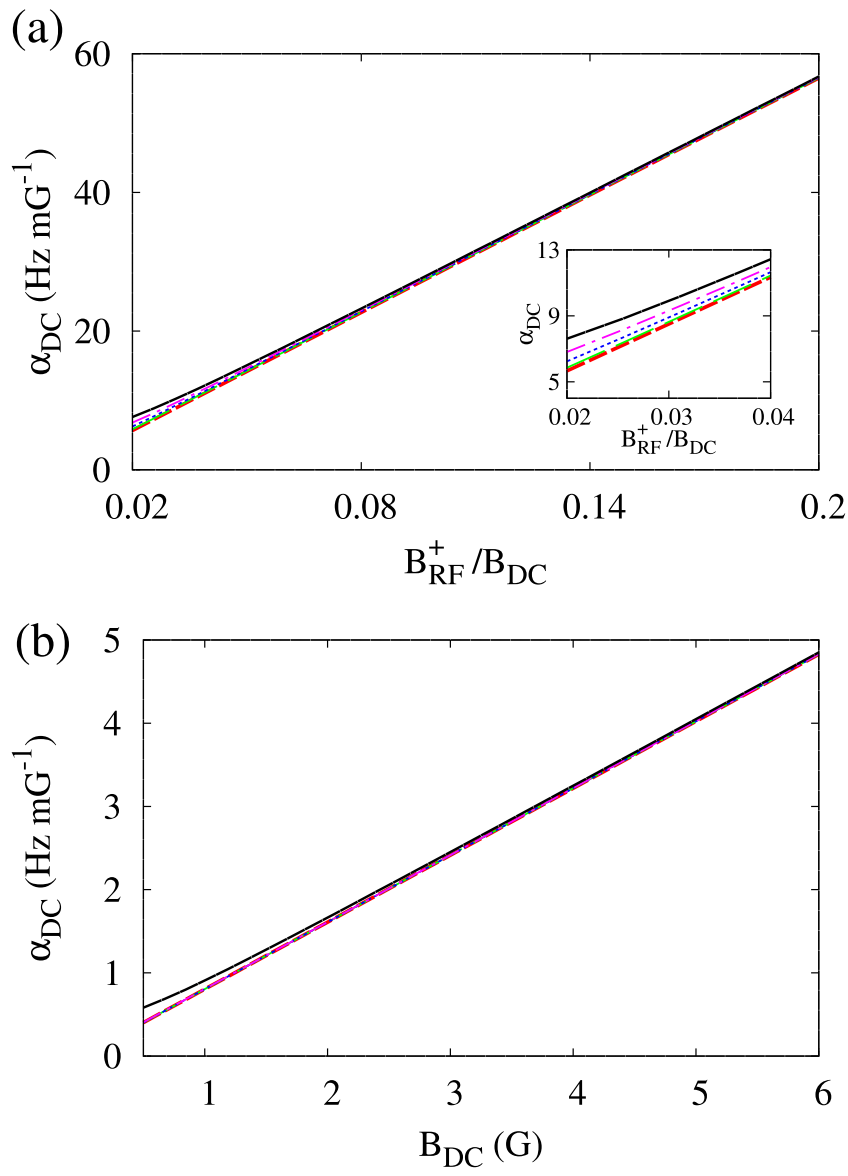

FIG. 8. Average sensitivity $\alpha_{\mathrm{DC}}$ of the dressed atomic transitions with respect to the static field [Eq. (17)], calculated using the field configuration optimized numerically. Panel (a) shows $\alpha_{\mathrm{DC}}$ as a function of the ratio $B_{\mathrm{RF}}^{+} / B_{\mathrm{DC}}$ for the DC fields $0.5 \mathrm{G}$ (short-dashed red), $1.70 \mathrm{G}$ (dashed green), 3.08 G (dotted blue), $4.28 \mathrm{G}$ (dot-dashed cyan), and $6.0 \mathrm{G}$ (solid black). Panel (b) shows $\alpha_{\mathrm{DC}}$ as a function of the static field, $B_{\mathrm{DC}}$, for RF fields of amplitude $0.5 \mathrm{G}$ (short-dashed red), $1.70 \mathrm{G}$ (dashed green), $3.08 \mathrm{G}$ (dotted blue), $4.28 \mathrm{G}$ (dot-dashed cyan), and $6.0 \mathrm{G}$ (solid black).

demonstrated in Ref. [35] for the case of atoms trapped in a magnetic quadrupole field. The two circular components can be produced by using two pairs of Helmholtz coils that point along the $x$ and $y$ directions, respectively. Each pair of coils is driven with an RF signal that contains both frequencies, with the appropriate phases (for details, see Ref. [35]).

\section{CONCLUSION}

Using an ultracold atomic cloud of ${ }^{87} \mathrm{Rb}$ in an optical dipole trap, we showed characteristic features of transitions between RF dressed states of the ground-state hyperfine manifolds. First, considering the trio of transitions between dressed states $|1, \bar{m}=-1\rangle \rightarrow\{|2, \bar{m}=0\rangle, \mid 2, \bar{m}=$ $1\rangle,|2, \bar{m}=2\rangle\}$, we observe a quadratic dependence of their transition frequencies as functions of total magnetic field $B_{\mathrm{DC}}$, with a significantly weaker curvature when $\Delta \bar{m}=0$. We found that our measurements can be explained quantitatively only after taking into account nonlinear Zeeman shifts and

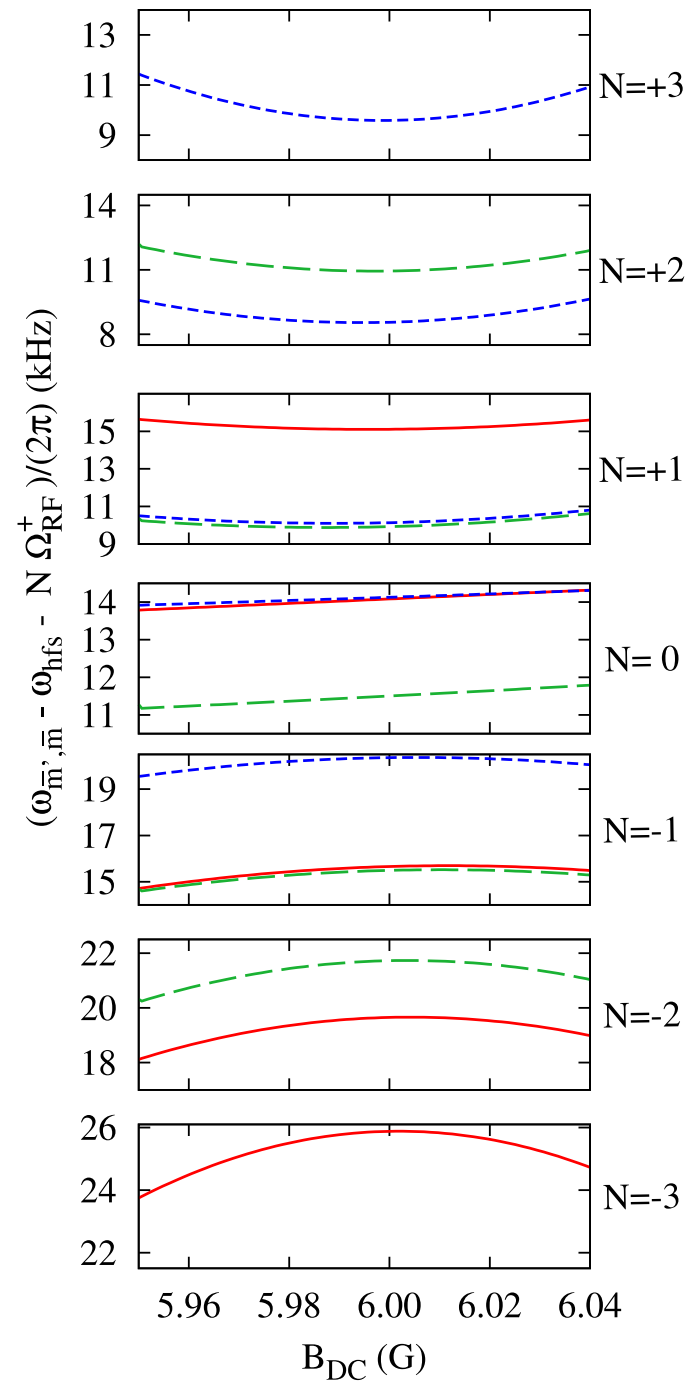

FIG. 9. Detuning of the resonant frequencies of all hyperfine transitions in RF dressed ${ }^{87} \mathrm{Rb}$, as functions of the static magnetic field. The field configuration is numerically optimized to minimize the rms of the linear sensitivity of all bichromatic dressed transitions (see text). In all cases $B_{\mathrm{DC}}^{0}=6.0 \mathrm{G}$ and the $B_{\mathrm{RF}}^{+}=0.12 \mathrm{G}$. Solid, dashed, and short-dashed lines correspond to transitions with the initial states $|F=1, \bar{m}\rangle=|1,-1\rangle,|1,0\rangle,|1,1\rangle$, respectively. The labels on the right-hand side indicate the final states as $\mid F=2, \bar{m}^{\prime}=$ $N-\bar{m}\rangle$.

beyond-RWA effects. However, a good qualitative description of the observed quadratic differential energy shifts can be obtained using well-known expressions for the dressed energies valid in the regimes of linear Zeeman shift and RWA.

We also study the coherence and linewidth of the transitions between RF-dressed states $|1, \bar{m}=-1\rangle \rightarrow|2, \bar{m}=1\rangle$ and $|1, \bar{m}=-1\rangle \rightarrow|2, \bar{m}=2\rangle$, as functions of the applied static field. We observe a significant increase in the decay time of Ramsey-type fringes for the transition $|1, \bar{m}=-1\rangle \rightarrow$ $|2, \bar{m}=1\rangle$ at a particular "magic" point. Following the same method, we observed a significant reduction of the linewidth of the transition $|1, \bar{m}=-1\rangle \rightarrow|2, \bar{m}=2\rangle$, reaching fluctuations of the order $\Delta v / v \approx 10^{-8}$. These experimental results demonstrate how monochromatic RF dressing can be tuned 
TABLE I. Comparison of the fractional frequency fluctuations $\left(\Delta \omega_{\bar{m}^{\prime}, \bar{m}} / \omega_{\bar{m}^{\prime}, \bar{m}}\right)$ for the transitions from the state $|1,-1\rangle$ to all final states (FS) of the $F=2$ manifold of Zeeman sublevels [see Eq. (19)]. The transition frequencies and sensitivities are calculated for transitions between (A) bare states, (B) monochromatic RF dressed states, and bichromatic RF dressed states with $\omega_{\mathrm{RF}}^{ \pm}(\mathrm{C})$ given by the RWA [Eqs. (13) and (14)] and (D) corrected to minimize $\alpha_{\mathrm{DC}}$, as shown in Fig. 7. In all cases, the field fluctuations are $\Delta B=0.1 \mathrm{mG}$. In (B) the dressing fiield is $B_{\mathrm{RF}}=0.2 B_{\mathrm{DC}}$, while in (C) and (D) we use $B_{\mathrm{RF}}^{+}=0.1 B_{\mathrm{DC}}$. In (D), $\Delta \omega_{\mathrm{RF}}^{\ell} / \omega_{\mathrm{RF}}^{\ell}=2.46 \times 10^{-3}$ and $2.50 \times 10^{-3}$ for $\ell=+$ and - , respectively.

\begin{tabular}{cccccc}
\hline \hline$B_{\mathrm{DC}}$ & FS & $\mathrm{A}$ & $\mathrm{B}$ & $\mathrm{C}$ & $\mathrm{D}$ \\
\hline \multirow{4}{*}{0.5} & $|2,-2\rangle$ & $2.7 \times 10^{-7}$ & $5.0 \times 10^{-9}$ & $6.8 \times 10^{-9}$ & $3.3 \times 10^{-11}$ \\
& $|2,-1\rangle$ & $1.8 \times 10^{-7}$ & $4.5 \times 10^{-9}$ & $4.5 \times 10^{-9}$ & $4.6 \times 10^{-11}$ \\
& $|2,0\rangle$ & $9.2 \times 10^{-8}$ & $4.0 \times 10^{-9}$ & $2.2 \times 10^{-9}$ & $6.6 \times 10^{-11}$ \\
& $|2,1\rangle$ & $3.1 \times 10^{-10}$ & $3.6 \times 10^{-9}$ & $2.0 \times 10^{-11}$ & $6.6 \times 10^{-11}$ \\
& $|2,2\rangle$ & $9.1 \times 10^{-8}$ & $3.2 \times 10^{-9}$ & $2.2 \times 10^{-9}$ & $2.3 \times 10^{-11}$ \\
& $|2,-2\rangle$ & $2.7 \times 10^{-7}$ & $4.8 \times 10^{-9}$ & $6.7 \times 10^{-9}$ & $1.9 \times 10^{-10}$ \\
& $|2,-1\rangle$ & $1.8 \times 10^{-7}$ & $4.2 \times 10^{-9}$ & $4.2 \times 10^{-9}$ & $3.5 \times 10^{-10}$ \\
3.2 & $|2,0\rangle$ & $9.1 \times 10^{-8}$ & $3.7 \times 10^{-9}$ & $1.8 \times 10^{-9}$ & $4.2 \times 10^{-10}$ \\
& $|2,1\rangle$ & $4.0 \times 10^{-12}$ & $3.3 \times 10^{-9}$ & $3.4 \times 10^{-10}$ & $3.7 \times 10^{-10}$ \\
& $|2,2\rangle$ & $9.1 \times 10^{-8}$ & $3.0 \times 10^{-9}$ & $2.3 \times 10^{-9}$ & $1.7 \times 10^{-10}$ \\
& $|2,-2\rangle$ & $2.7 \times 10^{-7}$ & $4.7 \times 10^{-9}$ & $6.5 \times 10^{-9}$ & $4.0 \times 10^{-10}$ \\
& $|2,-1\rangle$ & $1.8 \times 10^{-7}$ & $3.8 \times 10^{-9}$ & $3.7 \times 10^{-9}$ & $7.9 \times 10^{-10}$ \\
7.0 & $|2,0\rangle$ & $9.1 \times 10^{-8}$ & $3.2 \times 10^{-9}$ & $1.3 \times 10^{-9}$ & $9.2 \times 10^{-10}$ \\
& $|2,1\rangle$ & $4.2 \times 10^{-10}$ & $2.9 \times 10^{-9}$ & $7.8 \times 10^{-10}$ & $7.9 \times 10^{-10}$ \\
& $|2,2\rangle$ & $9.1 \times 10^{-8}$ & $2.8 \times 10^{-9}$ & $2.5 \times 10^{-9}$ & $3.8 \times 10^{-10}$ \\
\hline \hline & & & & & \\
\hline
\end{tabular}

to produce pairs of selected transitions protected against DCfield noise. To further reduce the transition linewidth to the order of $\mathrm{Hz}$ with this scheme, the fluctuations of the amplitude of the RF fields need to be stabilized at the level of order $10 \mu \mathrm{G}$. Recent reports using one [23], two [35], three [47], and four [48] RF-frequency dressing components also find this to be a limiting factor, and indicate that active control of the RF-field amplitude is therefore necessary [49].

Furthermore, we propose a bichromatic RF-dressing configuration to reduce the global sensitivity of the dressed atom to noise in the static field. We demonstrate that by independently tuning the frequencies of the two circular components of the RF field, it is possible to reduce the average linear DC sensitivity to the level of $\mathrm{Hz} \mathrm{mG}^{-1}$. This dressing scheme enables the protection at arbitrary DC-magnetic fields of up to $(2 F+1) \times\left(2 F^{\prime}+1\right)=15$ atomic microwave transitions in RF-dressed ${ }^{87} \mathrm{Rb}$, only limited by the noise in the RF generator. Also, this bichromatic dressing configuration can stabilize more than just one single atomic transition, which is useful to define stable qdits with $d>2$. Such systems present advantages for applications in quantum metrology [50] and enhanced fault tolerance for quantum information [33,34]. In addition, since the energies of the dressed states can be tuned precisely, these dressed states are attractive also for applications in quantum simulations [51] and for hybrid quantum systems that include devices operating in the RF/MW regime (e.g., superconducting resonators, NV centers, and trapped ions). Further research will be directed to determine multiparametric magic configurations in the regime of strong RF dressing, where multi-level atomic transitions can be made less sensitive to noise in DC and ac fields.
The techniques presented in this work are aimed at situations where the spatial extent of the atomic ensemble is limited (e.g., to $\sim 100$ s microns), such as in atom interferometry based on spin-dependent manipulation of ultracold atom clouds in adiabatic traps $[18,25,30,44,47]$. In many of these situations, the amplitude of the fluctuations of the transition frequencies of interest depends on the relative stability of the magnetic field, which can be enhanced with passive and active shielding techniques. Notice, however, that because of its bulkiness and limitations on optical access, shielding techniques [11,12] are better suited for applications with free atomic ensembles (i.e., not trapped) and working with intrinsically robust transitions (e.g., the $\left|F=I-1 / 2, m_{F}=0\right\rangle \rightarrow$ $\left|F=I+1 / 2, m_{F}=0\right\rangle$ of the electronic ground manifold of alkali atoms; see Appendix D). In contrast, dynamical decoupling and RF-dressing focus on manipulating the response of the atoms to fluctuations of the magnetic environment and, as we demonstrate here, allows us to synthesise robust transitions. These two techniques are therefore complementary and, indeed, RF-dressed protected states can become helpful to reduce the thickness- and thus the weight and cost-of magnetic shields.

Additionally, the use of a nondestructive imaging system (see, e.g., Reference [52]) allows real-time interrogation of the spin-dynamics of the system, which may become useful in both determining amplitude and polarization drifts and noise in a fast frequency range - at the timescale of the Rabi frequency-compared to the current single-shot measurement rate, which is limited by the experiment cycle time at the timescale of $10 v \mathrm{vs}$. [53].

The datasets generated for this paper are available in Ref.

\section{ACKNOWLEDGMENTS}

This work is supported by the project "HELLAS-CH" (MIS 5002735) which is implemented under the "Action for Strengthening Research and Innovation Infrastructures," funded by the Operational Programme "Competitiveness, Entrepreneurship and Innovation" (NSRF 2014-2020) and cofinanced by Greece and the European Union (European Regional Development Fund). We acknowledge financial support from the Greek Foundation for Research and Innovation (ELIDEK) in the framework of project, Guided Matter-Wave Interferometry under Grant No. 4823 and General Secretariat for Research and Technology (GSRT). G.V. received funding from the European Union's Horizon 2020 research and innovation programme under the Marie Skodowska-Curie Grant No. 750017. This work has been supported by UK EPSRC Grant No. EP/M013294/1 and the University of Sussex. The authors acknowledge the contribution of the COST Action CA16221.

G.A.S.-L. and H.M. contributed equally. H.M. and G.A.S.L. conceived and developed the main ideas of the paper. H.M. carried out the experimental measurements. H.M., S.P., G.V., and W.v.K. built the experimental setup. G.A.S.-L. and H.M. performed the data analysis. G.A.S.-L. was responsible for the theoretical and numerical work. B.M.G. and W.v.K. supervised the development of the work. All authors contributed to the result discussion and paper writing. 


\section{APPENDIX A: DRESSED STATES IN THE RWA}

In the main text, Eq. (4) defines the unitary transformation, $U_{F}$, between the bare and dressed basis as:

$$
\langle F, m \mid \bar{F}, \bar{m}\rangle=\sum_{n} e^{i n \omega_{\mathrm{RF}} t} U_{F, m ; \bar{F}, \bar{m}}^{n} .
$$

The Fourier coefficients of this expansion are determined by the Schrödinger equation and the condition Eq. (3), which translates into:

$$
U_{F}^{\dagger}(t)\left[H(t)-i \hbar \partial_{t}\right] U_{F}(t)=\sum_{\bar{F}, \bar{m}} \bar{E}_{\bar{F}, \bar{m}}|\bar{F}, \bar{m}\rangle\langle\bar{F}, \bar{m}| .
$$

This last expression gives us a straightforward physical interpretation of the dressed basis: the dressed states are the eigenenergy states observed in a frame of reference where the Hamiltonian is time independent. This concept is commonly used in quantum physics in the figure of RWA, where, after moving to a rotating frame of reference the time dependence of the Hamiltonian either cancels completely or can be neglected following perturbative arguments [41].

In the present case, we consider oscillating fields with a frequency comparable to the Zeeman splitting induced by a static magnetic field, but much smaller than the hyperfine splitting $\left(\hbar \omega_{\mathrm{RF}} \approx \mu_{B} g_{F} B_{\mathrm{DC}} \ll \Delta E_{\mathrm{Hyperfine}}\right)$. Under these conditions, we can neglect the intermanifold coupling and apply the RWA within each hyperfine manifold, where the transformation to the dressed basis can be written as a combination of rotations in the space of angular momentum [23]:

$$
U_{F}(t)=e^{i \theta_{y} F_{y}} e^{-i \frac{g_{F}}{\left|g_{F}\right|} \omega_{\mathrm{RF}} t F_{z}},
$$

with

$$
\tan \left(\theta_{y}\right)=\frac{\sqrt{2}\left|\Omega_{\mathrm{RF}}^{\operatorname{sgn}\left(g_{F}\right)}\right|}{\omega_{0}-\omega_{\mathrm{RF}}},
$$

where $\hbar \omega_{0}=\left|\mu_{B} g_{F} B_{\mathrm{DC}}\right|$ defines the Larmor frequency and the Rabi frequency $\left|\Omega_{\mathrm{RF}}^{\mathrm{sgn}\left(g_{F}\right)}\right|$ is defined in Eq. (6). The corresponding dressed energies are

$$
\bar{E}_{F, \bar{m}}=E_{F}+\operatorname{sgn}\left(g_{F}\right) \bar{m} \sqrt{\left(\hbar \omega_{0}-\hbar \omega_{\mathrm{RF}}\right)^{2}+2\left|\hbar \Omega_{\mathrm{RF}}^{\mathrm{sgn}\left(g_{F}\right)}\right|^{2}},
$$

where $E_{F}=A[F(F+1)-I(I+1)-J(J+1)] / 2$ is the hyperfine splitting. This dressed energy leads to the dependence of the resonant condition with respect to the field configuration:

$$
\begin{aligned}
\omega_{\mathrm{MW}}= & n \omega_{\mathrm{RF}}+\frac{(I+1 / 2) A}{\hbar} \\
& +\frac{g_{F+1}}{\left|g_{F+1}\right|} \bar{m}^{\prime} \sqrt{2}\left|\Omega_{\mathrm{RF}}^{+}\right|-\frac{g_{F}}{\left|g_{F}\right|} \bar{m} \sqrt{2}\left|\Omega_{\mathrm{RF}}^{-}\right| \\
& +\left(\frac{g_{F+1}}{\left|g_{F+1}\right|} \frac{\bar{m}^{\prime}}{\left|\Omega_{\mathrm{RF}}^{+}\right|}-\frac{g_{F}}{\left|g_{F}\right|} \frac{\bar{m}}{\left|\Omega_{\mathrm{RF}}^{-}\right|}\right) \frac{\omega_{\mathrm{RF}}^{2}}{2^{3 / 2}} \\
& -\left(\frac{\bar{m}^{\prime} g_{F+1}}{\left|\Omega_{\mathrm{RF}}^{+}\right|}-\frac{\bar{m} g_{F}}{\left|\Omega_{\mathrm{RF}}^{-}\right|}\right) \omega_{\mathrm{RF}} \frac{\mu_{B} B_{\mathrm{DC}}}{\sqrt{2} \hbar}+\frac{1}{2^{3 / 2}} \\
& \times\left[\frac{g_{F+1}}{\left|g_{F+1}\right|} \frac{\bar{m}^{\prime} g_{F+1}^{2}}{\left|\Omega_{\mathrm{RF}}^{+}\right|}-\frac{g_{F}}{\left|g_{F}\right|} \frac{\bar{m} g_{F}^{2}}{\left|\Omega_{\mathrm{RF}}^{-}\right|}\right]\left(\frac{\mu_{B} B_{\mathrm{DC}}}{\hbar}\right)^{2},
\end{aligned}
$$

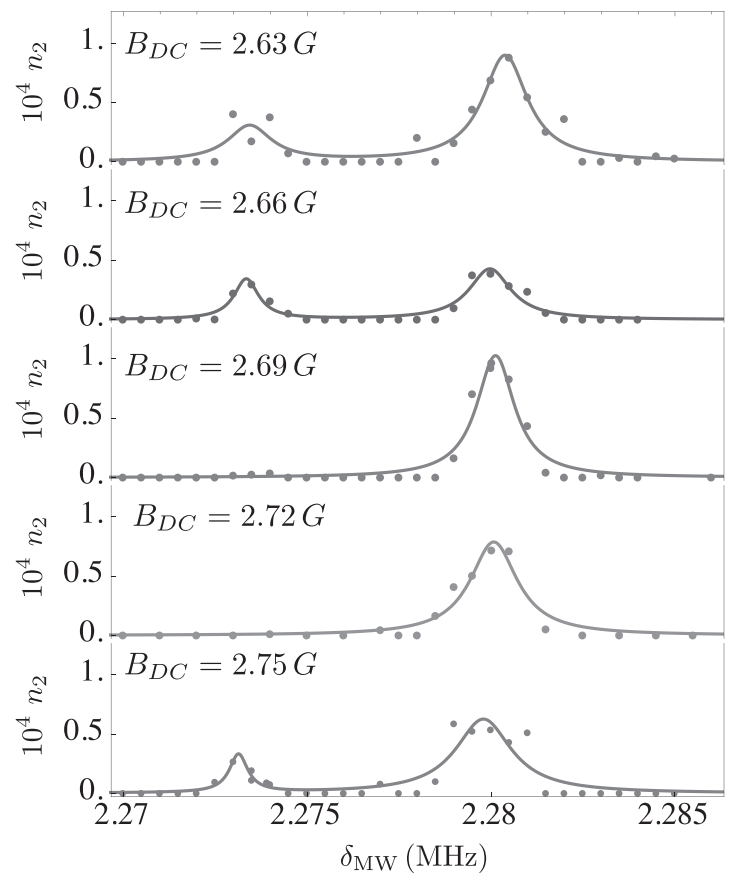

FIG. 10. Spectra for selected values of DC field in Fig. 4(a). From top to bottom, $B_{\mathrm{DC}}=2.63 \mathrm{G}, 2.66 \mathrm{G}, 2.69 \mathrm{G}, 2.72 \mathrm{G}$, and $2.75 \mathrm{G}$. The Rabi frequency is $\Omega_{\mathrm{RF}} / 2 \pi \mathrm{kHz}$ and the RF frequency is $\omega_{\mathrm{RF}} / 2 \pi=2.27 \mathrm{MHz}$. Just like in the rest of the text, $n_{2}$ is the atom number measured with absorption imaging in $F=2$. Each point corresponds to one measurement. We fit Lorentzian curves for each observed peak. We observe the intermittent appearance of a second weaker peak due to transitions from atoms that did not adiabatically follow the dressing sequence.

which is valid for intermanifold transitions, $|F, \bar{m}\rangle \rightarrow \mid F+$ $\left.1, \bar{m}^{\prime}\right\rangle$ and in the vicinity of the condition of resonant RF dressing, $\mu_{B}\left|g_{F} B_{\mathrm{DC}}\right| \approx \hbar \omega_{\mathrm{RF}}$.

This formulation can be easily extended to situations with polychromatic driving. The time-evolution operator should be then expressed as a multidimensional Fourier series with as many dimensions as the number of applied fields with incommensurately frequencies. More explicitly, the dressed state defined in Eq. (A1) becomes

$$
\langle F, m \mid \bar{F}, \bar{m}\rangle=\sum_{\vec{n}} e^{i \vec{n} \vec{\omega} t} U_{F, m ; \bar{F}, \bar{m}}^{\vec{n}},
$$

with $\vec{\omega}=\left(\omega_{1}, \omega_{2}, \ldots\right)$ as the vector formed with all the applied frequencies and $\vec{n}$ a vector with integer components. After plugging this ansatz in the Schrödinger equation Eq. (A2) we obtain a standard eigenproblem defining the coefficients $U_{F, m ; \bar{F}, \bar{m}}^{\vec{n}}$ and generalized dressed energies $\bar{E}_{\bar{F}, \bar{m}}$.

\section{APPENDIX B: MICROWAVE SPECTROSCOPY OF THE DRESSED TRANSITION $|1,-1\rangle \rightarrow|2,1\rangle$ NEAR THE TWO-PHOTON CONDITION}

We measured the line shift of the transition $|1, \bar{m}=-1\rangle \rightarrow$ $|2, \bar{m}=1\rangle$ in Fig. 4(a). We intermittently observed a second weaker peak, with some examples in Fig. 10. Here we interrogate the first group of transitions, i.e., those with $N=1$, following Eq. (9), with $\omega_{\mathrm{RF}} / 2 \pi=2.27 \mathrm{MHz}$. A qualitative 
analysis indicates that the each peak is produced by atoms in different dressed states, with the intensity of each corresponding to their population. This second resonance indicates that some atoms do not follow adiabatically our dressing sequence [45].

\section{APPENDIX C: OFF-RESONANT CORRECTIONS TO THE RWA AND LINEAR SENSITIVITY OF THE BICHROMATIC-DRIVEN ATOM TO RF AMPLITUDE NOISE}

The susceptibility of the dressed transitions with respect to variations of the dressing parameters can be calculated correcting the RWA dressed energy by including perturbative shifts of the Zeeman states:

$$
\begin{aligned}
\bar{E}_{F, \bar{m}}= & E_{F}+\frac{g_{F}}{\left|g_{F}\right|} \bar{m} \\
& \times \sqrt{\left(\mu_{B}\left|g_{F}\right| B_{\mathrm{DC}}-\hbar \omega_{\mathrm{F}}+\hbar \Delta_{F}\right)^{2}+2 \hbar\left|\Omega_{\mathrm{RF}}^{\mathrm{sgn}\left(g_{F}\right)}\right|^{2}}
\end{aligned}
$$

with $E_{F}=A[F(F+1)-I(I+1)-J(J+1)] / 2$ and where $\Delta_{F}$ has contributions from the fields which are counterrotating in the dressed frame of reference. The total shift can be approximated by:

$$
\hbar \Delta_{F}=\frac{1}{2} \frac{g_{F}}{\left|g_{F}\right|} \frac{\left|\mu_{B} g_{F}\left[B_{\mathrm{RF}}^{-\operatorname{sgn}\left(g_{F}\right)}+\Delta B_{\mathrm{RF}}^{\operatorname{sgn}\left(g_{F}\right)}\right]\right|^{2}}{\mu_{B}\left|g_{F}\right| B_{\mathrm{DC}}+\hbar \omega_{0}},
$$

where $\omega_{0}=\left(\omega_{+}+\omega_{-}\right) / 2$ and $\Delta B_{\mathrm{RF}}^{\ell}$ is the counter-rotating component of the field oscillating at frequency $\omega_{\ell}$.

Another important contribution to the broadening of the transition lines is their instability with respect to variations in the amplitude of the dressing fields. In this case, we can distinguish four contributions emerging from the decomposition of the variations of each dressing frequency into $\sigma_{+}$ and $\sigma_{-}$polarizations. To evaluate the effects of fluctuations of the RF field, we split the noise of each polar component of the RF field into corotating and counter-rotating contributions:

$$
B_{\mathrm{RF}}^{\ell}=B_{\mathrm{RF}, 0}^{\ell}+\delta B_{\mathrm{RF}, \ell}^{\ell}+\delta B_{\mathrm{RF},-\ell}^{\ell},
$$

where $\ell \in+,-, B_{\mathrm{RF}, 0}^{\ell}$ is the rms value of the field, and $\delta B_{\mathrm{RF},(-) \ell}^{\ell}$ is the component of the fluctuation corotating (counter-rotating) with the $\sigma_{\ell}\left(\sigma_{-\ell}\right)$ component of the RF field. As in Sec. IV, we quantify these effects by defining average linear sensitivities, $\alpha_{\mathrm{RF}, m}^{\ell}$ for each component of the ( $m \in\{+,-\}$ ) of the two dressing fields $\ell \in\{+,-\}$ :

$$
\alpha_{\mathrm{RF}, \ell}^{m}=\sqrt{\frac{1}{15} \sum_{m=-1}^{1} \sum_{m^{\prime}=-2}^{2}\left(\frac{\partial \omega_{\bar{m}, \bar{m}^{\prime}}}{\partial \delta B_{\mathrm{RF}, m}^{\ell}}\right)^{2}}
$$

In Fig. 11 we show the atomic sensitivity associated with the corotating noisy components of the dressing fields $(m=$ $\ell$ ), when using the optimized frequency configuration. The corotating noise modifies the amplitude of the dressing field, leading to a sensitivity that weakly depends on the static field.

Another effect due to noise of the dressing field comes from the counter-rotating noisy component, which causes offresonant energy shifts similar to the ones described before in Eq. (C1), but this time oscillating at the same frequency of the
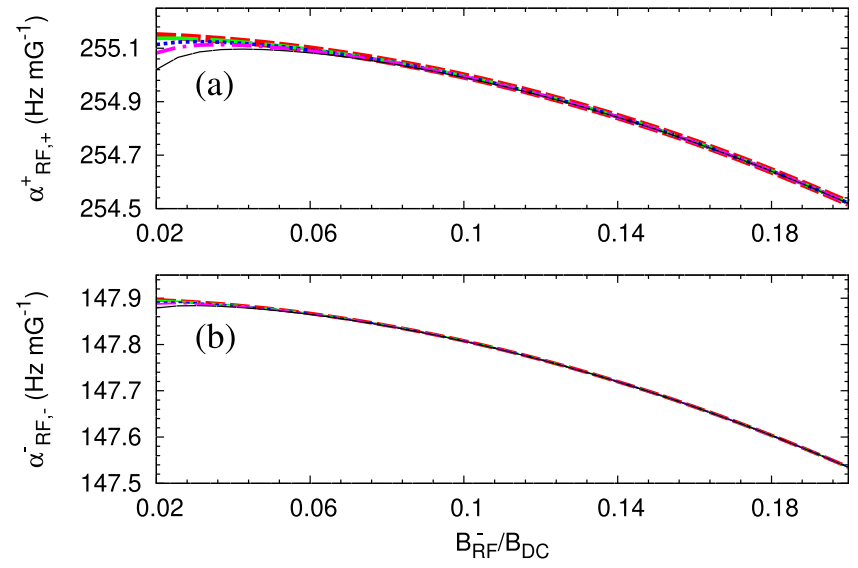

FIG. 11. The rms susceptibility of the dressed transition frequencies of ${ }^{87} \mathrm{Rb}$ with respect to corotating variations of the (a) $\sigma_{+}$and (b) $\sigma_{-}$dressing fields as functions of the dressing field amplitude. The static field are $0.5 \mathrm{G}$ (dashed line), $1.5 \mathrm{G}$ (long-dashed line), 3.0 G (dotted line), $4.5 \mathrm{G}$ (dashed-dotted line), and 6.0 G (solid line). For each dressing configuration, we evaluate the optimal combination of $\sigma_{ \pm}$frequencies that minimize $\alpha_{\text {DC }}$ Eq. (17).

corresponding dressing component. In Fig. 12 we show the a rms linear susceptibility of all transitions, $\alpha_{\mathrm{RF},-\ell}^{\ell}$, induced by counter-rotating variations of the dressing fields, using the frequency configuration that minimize the sensitivity to static fields.

The scaling and order of magnitude of $\alpha_{\mathrm{RF}, \ell}^{m}$ can be obtained by calculating second-order perturbative energy shifts and applying the RWA (see Appendix B). Note that the sensitivity to variations of the dressing fields is two orders of magnitude larger than the sensitivity to variations of the static field with the optimized parameters (compare with Fig. 8). To reduce the line broadening of resonant transitions to the level of a few $\mathrm{Hz}$, the fluctuations of dressing RF fields should
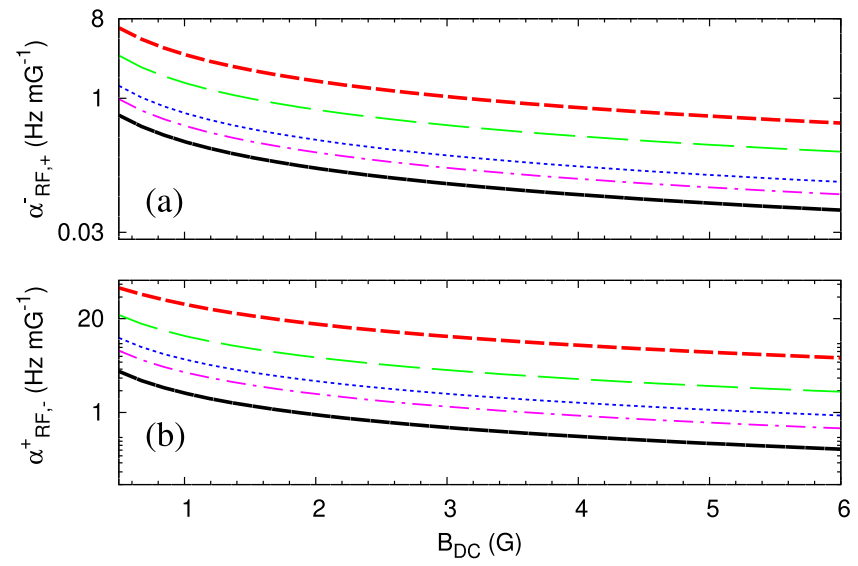

FIG. 12. The rms linear sensitivity of the dressed transition frequencies of ${ }^{87} \mathrm{Rb}$ with respect to the counter-rotating polar components (a) $\sigma_{+}$and (b) $\sigma_{-}$of the variations of the dressing fields $\sigma_{-}$(a) and $\sigma_{+}$(b). The scaled amplitudes of the dressing fields are $0.02 B_{\mathrm{DC}}$ (dashed line), $0.03 B_{\mathrm{DC}}$ (long-dashed line), $0.1 B_{\mathrm{DC}}$ (dotted line), $0.15 B_{\mathrm{DC}}$ (dashed-dotted line), and $0.2 B_{\mathrm{DC}}$ (solid line). For each dressing configuration, we evaluate the optimal combination of $\sigma_{ \pm}$frequencies that minimize $\alpha_{\mathrm{DC}}$. 
of order $\approx 10 \mu \mathrm{G}$, which corresponds to a relative amplitude fluctuation of the order $10^{-5}-10^{-6}$.

\section{APPENDIX D: COMPARISON WITH OTHER TECHNIQUES}

In our experiment, we optimized the RF-dressing configuration and observed fractional frequency fluctuations of $\Delta \omega_{1,-1} / \omega_{1,-1} \approx 8.6 \times 10^{-9}$ [see Fig. 4(b)]. Our measurements are consistent with an effective suppression of effects due to variations of the static magnetic field, with the remaining fluctuations due to contributions from other experimental variables. Furthermore, our theoretical analysis demonstrates that by tuning a bichromatic RF-dressing field to attain a global optimum over all the hyperfine transitions in the manifold, it would be possible to reach fractional frequency fluctuations of order $10^{-11}$. This level of fluctuations would be that associated with variations of the static field with a relative stability of $10^{-4}$ (see Table I).

These results can be compared with observations of frequency fluctuations for the transition between the states $\mid F=$ $\left.1, m_{F}=-1\right\rangle$ and $\left|F=2, m_{F}=1\right\rangle$ of ${ }^{87} \mathrm{Rb}$. Szmuk et al. [18] demonstrates shot-to-shot frequency fluctuations of order $\sim 10^{-13}$ in a well-shielded environment that provides a relative stability of the DC magnetic field of $10^{-6}$. Kazakov and Schumm [20] explore theoretically the use of off-resonant RF dressing for protection against variations of the amplitude of the DC and RF fields. For a typical optimal configuration, they estimate that the fractional frequency fluctuations due to variations of the DC field are of order $10^{-12}$ for a relative stability of $10^{-4}$ (see Fig. 6a in Ref. [20]). Finally, Sárkány et al. [19] explore theoretically and experimentally the use of off-resonant MW dressing in a shielded environment and find fractional frequency fluctuations of the order of $10^{-12}$ for a DC relative stability of $10^{-4}$ (see Fig. 3 in Ref. [19]).
[1] B. Bloom, T. Nicholson, J. Williams, S. Campbell, M. Bishof, X. Zhang, W. Zhang, S. Bromley, and J. Ye, Nature 506, 71 (2014).

[2] I. Baumgart, J.-M. Cai, A. Retzker, M. B. Plenio, and C. Wunderlich, Phys. Rev. Lett. 116, 240801 (2016).

[3] R. Zhao, Y. Dudin, S. Jenkins, C. Campbell, D. Matsukevich, T. Kennedy, and A. Kuzmich, Nat. Phys. 5, 100 (2009).

[4] J. Preskill, Proc. R. Soc. A 454, 385 (1998).

[5] D. Gottesman, Chaos Solitons Fract. 10, 1749 (1999).

[6] G. A. Sinuco-León and B. M. Garraway, New J. Phys. 18, 035009 (2016).

[7] G. Santarelli, P. Laurent, P. Lemonde, A. Clairon, A. G. Mann, S. Chang, A. N. Luiten, and C. Salomon, Phys. Rev. Lett. 82, 4619 (1999).

[8] F. Meier and D. Loss, Phys. Rev. B 71, 094519 (2005).

[9] D. A. Golter and H. Wang, Phys. Rev. Lett. 112, 116403 (2014).

[10] T. D. Ladd, D. Maryenko, Y. Yamamoto, E. Abe, and K. M. Itoh, Phys. Rev. B 71, 014401 (2005).

[11] S. Dickerson, J. M. Hogan, D. M. Johnson, T. Kovachy, A. Sugarbaker, S.-w. Chiow, and M. A. Kasevich, Rev. Sci. Instrum. 83, 065108 (2012).

[12] L. Botti, R. Buffa, A. Bertoldi, D. Bassi, and L. Ricci, Rev. Sci. Instrum. 77, 035103 (2006).

[13] L. Viola and E. Knill, Phys. Rev. Lett. 90, 037901 (2003).

[14] J. Cai, B. Naydenov, R. Pfeiffer, L. P. McGuinness, K. D. Jahnke, F. Jelezko, M. B. Plenio, and A. Retzker, New J. Phys. 14, 113023 (2012).

[15] A. Bermudez, P. O. Schmidt, M. B. Plenio, and A. Retzker, Phys. Rev. A 85, 040302(R) (2012).

[16] A. Laucht, R. Kalra, S. Simmons, J. P. Dehollain, J. T. Muhonen, F. A. Mohiyaddin, S. Freer, F. E. Hudson, K. M. Itoh, D. N. Jamieson et al., Nat. Nanotechnol. 12, 61 (2017).

[17] N. Timoney, I. Baumgart, M. Johanning, A. Varón, M. B. Plenio, A. Retzker, and C. Wunderlich, Nature 476, 185 (2011).

[18] R. Szmuk, V. Dugrain, W. Maineult, J. Reichel, and P. Rosenbusch, Phys. Rev. A 92, 012106 (2015).

[19] L. Sárkány, P. Weiss, H. Hattermann, and J. Fortágh, Phys. Rev. A 90, 053416 (2014).
[20] G. A. Kazakov and T. Schumm, Phys. Rev. A 91, 023404 (2015).

[21] H. Katori, M. Takamoto, V. G. Pal'chikov, and V. D. Ovsiannikov, Phys. Rev. Lett. 91, 173005 (2003).

[22] S. Haroche, C. Cohen-Tannoudji, C. Audoin, and J. P. Schermann, Phys. Rev. Lett. 24, 861 (1970).

[23] G. A. Sinuco-León, B. M. Garraway, H. Mas, S. Pandey, G. Vasilakis, V. Bolpasi, W. von Klitzing, B. Foxon, S. Jammi, K. Poulios, and T. Fernholz, Phys. Rev. A 100, 053416 (2019).

[24] B. M. Garraway and H. Perrin, J. Phys. B: At., Mol. Opt. Phys. 49, 172001 (2016).

[25] S. Pandey, H. Mas, G. Drougakis, P. Thekkeppatt, V. Bolpasi, G. Vasilakis, K. Poulios, and W. von Klitzing, Nature 570, 205 (2019).

[26] O. Morizot, L. Longchambon, R. K. Easwaran, R. Dubessy, E. Knyazchyan, P.-E. Pottie, V. Lorent, and H. Perrin, Eur. Phys. J. D 47, 209 (2008).

[27] G. Sinuco-León and B. M. Garraway, New J. Phys. 14, 123008 (2012).

[28] F. Bloch and A. Siegert, Phys. Rev. 57, 522 (1940).

[29] A. Smith, B. E. Anderson, H. Sosa-Martinez, C. A. Riofrío, I. H. Deutsch, and P. S. Jessen, Phys. Rev. Lett. 111, 170502 (2013).

[30] D. Trypogeorgos, A. Valdés-Curiel, N. Lundblad, and I. B. Spielman, Phys. Rev. A 97, 013407 (2018).

[31] G. Kucsko, S. Choi, J. Choi, P. C. Maurer, H. Zhou, R. Landig, H. Sumiya, S. Onoda, J. Isoya, F. Jelezko, E. Demler, N. Y. Yao, and M. D. Lukin, Phys. Rev. Lett. 121, 023601 (2018).

[32] S. Choi, N. Y. Yao, and M. D. Lukin, Phys. Rev. Lett. 119, 183603 (2017).

[33] E. T. Campbell, Phys. Rev. Lett. 113, 230501 (2014).

[34] B. P. Lanyon, M. Barbieri, M. P. Almeida, T. Jennewein, T. C. Ralph, K. J. Resch, G. J. Pryde, J. L. O'Brien, A. Gilchrist, and A. G. White, Nat. Phys. 5, 134 (2009).

[35] H. Mas, S. Pandey, G. Vasilakis, and W. von Klitzing, New J. Phys. 21, 123039 (2019).

[36] P. J. J. O’Malley, J. Kelly, R. Barends, B. Campbell, Y. Chen, Z. Chen, B. Chiaro, A. Dunsworth, A. G. Fowler, I.-C. Hoi, E. Jeffrey, A. Megrant, J. Mutus, C. Neill, C. Quintana, P. 
Roushan, D. Sank, A. Vainsencher, J. Wenner, T. C. White, A. N. Korotkov, A. N. Cleland, and J. M. Martinis, Phys. Rev. Appl. 3, 044009 (2015).

[37] S. Brouard and J. Plata, Phys. Rev. A 68, 012311 (2003).

[38] B. E. Anderson, H. Sosa-Martinez, C. A. Riofrío, I. H. Deutsch, and P. S. Jessen, Phys. Rev. Lett. 114, 240401 (2015).

[39] J. H. Shirley, Phys. Rev. 138, B979 (1965).

[40] P. Weinberg, M. Bukov, L. D’Alessio, A. Polkovnikov, S. Vajna, and M. Kolodrubetz, Phys. Rep. 688, 1 (2017).

[41] G. Series, Phys. Rep. 43, 1 (1978).

[42] G. A. Sinuco-León, SoftwareX 12, 100603 (2020).

[43] H. Mas, Ph.D. thesis, University of Crete, 2019.

[44] D. M. Harber, H. J. Lewandowski, J. M. McGuirk, and E. A. Cornell, Phys. Rev. A 66, 053616 (2002).

[45] K. A. Burrows, H. Perrin, and B. M. Garraway, Phys. Rev. A 96, 023429 (2017).

[46] When using the RWA conditions, we have $\frac{\partial \alpha_{\mathrm{DC}}}{\partial B_{\mathrm{DC}}} \approx 0$ and $\frac{\partial \alpha_{\mathrm{DC}}}{\partial B_{\mathrm{RF},+}}>0$. With the optimized RF frequencies $\frac{\partial \alpha_{\mathrm{DC}}}{\partial B_{\mathrm{DC}}}>0$ and $\frac{\partial \alpha_{\mathrm{DC}}}{\partial B_{\mathrm{RF},+}} \approx 0$.
[47] T. L. Harte, E. Bentine, K. Luksch, A. J. Barker, D. Trypogeorgos, B. Yuen, and C. J. Foot, Phys. Rev. A 97, 013616 (2018).

[48] E. Bentine, T. L. Harte, K. Luksch, A. J. Barker, J. Mur-Petit, B. Yuen, and C. J. Foot, J. Phys. B: At., Mol. Opt. Phys. 50, 094002 (2017).

[49] B. Merkel, K. Thirumalai, J. Tarlton, V. Schäfer, C. Ballance, T. Harty, and D. Lucas, Rev. Sci. Instrum. 90, 044702 (2019).

[50] V. Giovannetti, S. Lloyd, and L. Maccone, Nat. Photon. 5, 222 (2011).

[51] P. Doria, T. Calarco, and S. Montangero, Phys. Rev. Lett. 106, 190501 (2011).

[52] W. Dubosclard, S. Kim, and C. L. Garrido Alzar, Commun. Phys. 4, 35 (2021).

[53] G. A. Sinuco-Leon, H. Mas, S. Pandey, G. Vasilakis, B. M. Garraway, and W. von Klitzing, Data cite for "Decoherence-free radio-frequency-dressed subspaces", doi:10.25377/sussex.14770803 (2021). 